\title{
CHEMICALLY REACTING IONIZED RADIATIVE FLUID FLOW THROUGH AN IMPULSIVELY STARTED VERTICAL PLATE WITH INDUCED MAGNETIC FIELD
}

\author{
T. AHMED and Md. M. ALAM \\ Mathematics Discipline, Khulna University \\ Khulna 9208, BANGLADESH \\ M. FERDOWS \\ Research Group in Fluid Flow Modeling and Simulation \\ Department of Applied Mathematics, University of Dhaka \\ Dhaka 1000, BANGLADESH \\ E-mail: ferdows@du.ac.bd \\ E.E. TZIRTZILAKIS \\ Department of Mechanical Engineering \\ Technological Educational Institute of Western Greece \\ 26334 Patras, GREECE
}

\begin{abstract}
Numerical studies have been performed to examine the chemically reacting ionized fluid flow through a vertical plate with induced magnetic field. This study is performed for the cooling problem. To obtain the nondimensional non-similar momentum, the induced magnetic field, energy and concentration equations, usual nondimensional variables have been used. The numerical solutions for the velocity fields, induced magnetic fields, temperature distribution as well as concentration distribution are obtained for associated parameters using the explicit finite difference method. The local and average shear stresses, current densities, Nusselt number as well as the Sherwood number are also investigated. The obtained results are discussed with the help of graphs to observe effects of various parameters entering into the problem. Also the stability conditions of the explicit finite difference method are analyzed. Finally, a qualitative comparison of the present results with previously published results has been made.
\end{abstract}

Key words: boundary layer, radiative flow, strong magnetic field, ionized gas, flow fields.

\section{Introduction}

The effects of ionized fluid flow on MHD boundary layer flow have become important in several industrial processes, scientific and engineering fields. It has been found by Cowling [1] that the ionized fluid has two distinct effects. The first effect- electric currents can flow in an ionized fluid because of relative diffusion of the ionized gas and electrons due to agencies of electric forces. The second effect depends wholly on the magnetic field. The convection flow is often encountered in nuclear reactors or in the study of planets and stars. There are many engineering problems which are susceptible to ionized fluid flow analysis. It is useful in astrophysics because much of the universe is filled with widely spaced charged particles and permeated by magnetic fields and so the continuum assumption becomes applicable. In many engineering applications, combined heat and mass transfer play an important role in fluids condensing or boiling at a solid surface. The combined heat and mass transfer are due to buoyancy forces caused by thermal and mass diffusions. Condensing and boiling are characteristic for many separation processes in chemical engineering

\footnotetext{
* To whom correspondence should be addressed
} 
such as drying, evaporation, distillation, condensation, rectification and absorption of a fluid. The combined buoyancy effects of thermal and mass diffusions on natural convection flow have been investigated by Gebhart and Pera [2]. The studies of MHD incompressible viscous flows with Hall currents have grown considerably because of their engineering applications to the problems of Hall accelerators, MHD generators, constructions of turbines and centrifugal machines, as well as flight magnetohydrodynamics. The effects of Hall currents on free convective flow through a porous medium bounded by an infinite vertical plate have been studied by Ram [3], when a strong magnetic field is imposed in a direction which is perpendicular to the free stream and makes an angle to the vertical direction. The Hall effects on an unsteady MHD free convective heat and mass transfer flow through a porous medium near an infinite vertical porous plate with constant heat flux and variable suction have been analyzed by Sattar and Alam [4].

The interaction of buoyancy with thermal radiation has studied extensively greatly during the last decade due to its importance in many practical applications. The combined effects of gas radiation and magnetic field on the mixed convection flow from a permeable vertical plate have been investigated by Aydin and Kaya [5] with the extension of MHD mixed convection flow about a permeable vertical plate.

The Soret and Dufour effects have been found to influence the flow field in mixed convection boundary layer over a vertical surface embedded in a porous medium. The effect of thermal radiation, Hall currents, Soret and Dufour effects on MHD flow by mixed convection over a vertical surface in porous media has been studied by Shateyi et al. [6]. The effects of Soret, Dufour numbers, chemical reaction, thermal radiation and volumetric heat generation/absorption on mixed convection stagnation point flow on an isothermal vertical plate in porous media have been analyzed by Olanrewaju and Gbadeyan [7]. Recently, the effects of Soret and Dufour on unsteady MHD flow by mixed convection over a vertical surface in porous media with internal heat generation, chemical reaction and Hall current have been investigated by Aurangzaib and Shafie [8]. Ahmed and Alam [9] solved the Aurangzaib and Shafie [8] model by the implicit finite difference method.

All the above papers concentrated on MHD free convection heat and mass transfer flow of an incompressible viscous fluid past a continuously moving surface under only the action of a transverse magnetic field with or without thermal diffusion, thermo and chemical reaction for a small magnetic Reynolds number. If the magnetic Reynolds number of the flow is not taken to be small enough and the flow under the action of a strong magnetic field that induced another magnetic field is of great interest in geophysics and astrophysics. The steady combined heat and mass transfer flow with an induced magnetic field has been analytically analyzed by Chaudhary and Sharma [10]. The unsteady heat and mass transfer by mixed convection flow from a vertical porous plate with an induced magnetic field, constant heat and mass fluxes has been investigated by Alam et al. [11]. The numerical simulation and stability on MHD free convective heat and mass transfer unsteady flow through a porous medium in a rotating system with an induced magnetic field has been investigated by Haque et al. [12]. For an unsteady two dimensional case, with Hall effect, chemical reaction, thermal radiation, thermal-diffusion, diffusion thermo, induced magnetic field and inclined uniform magnetic field the above problem becomes more complicated. These types of problems play a special role in nature, in many separation processes such as isotope separation, in mixtures between gases, in many industrial applications such as solidification of binary alloy as well as in astrophysical and geophysical engineering.

Hence our aim of this research is to extend the work of Ahmed and Alam [9] with an induced magnetic field. The problem has been solved by the explicit finite difference method (Carnahan et al. [13]). The governing equations involved in this problem have been transformed into dimensionless non-similar coupled partial differential equations by usual transformations. Finally, a comparison of the present results with the results of Haque et al. [12]) has been made.

\section{Mathematical model of the flow}

A flow model of an unsteady chemically reacting ionized mixed convective heat and mass transfer flow of an electrically conducting incompressible viscous fluid past an electrically nonconducting semiinfinite vertical porous plate with thermal diffusion and diffusion thermo effect is considered. It 
considered that the effects of Joule heating, radiation and heat source or absorption are considered. The positive $x$ coordinate is measured along the plate in the direction of fluid motion and the positive $y$ coordinate is measured normal to the plate. The leading edge of the plate is taken as coincident with the $z-$ axis. Initially, it is considered that the plate as well as the fluid are at the same temperature $T\left(=T_{\infty}\right)$ and the concentration level $C\left(=C_{\infty}\right)$. Also, it is assumed that the fluid and the plate are at rest after that the plate is moving with a constant velocity in its own plane. Instantaneously at time $t>0$, the temperature of the plate and species concentration are raised to and $C_{w}\left(>C_{\infty}\right)$ respectively, which are thereafter maintained constant, where $T_{w}, C_{w}$ are temperature and species concentration at the wall and $T_{\infty}, C_{\infty}$ are the temperature and concentration of the species outside the plate respectively. The physical configuration of the problem is shown in Fig.1.

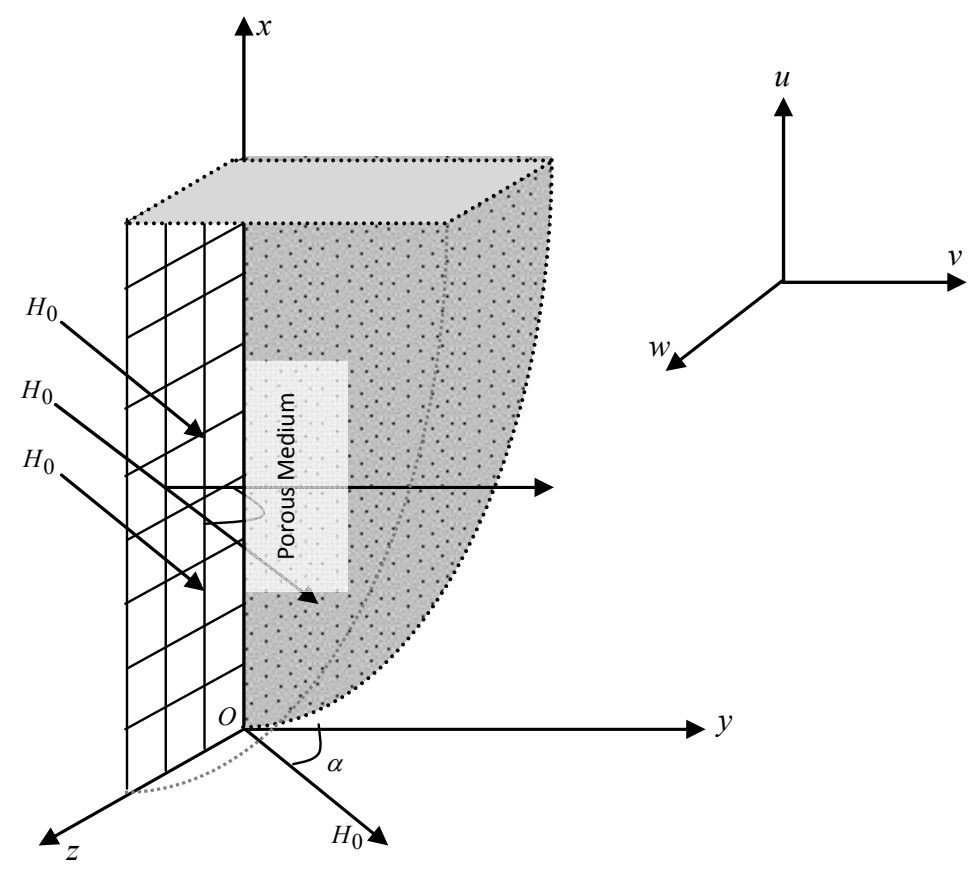

Fig.1. Geometrical configuration and coordinate system.

A strong uniform magnetic field $H_{0}$ can be taken as $\left(0, \lambda H_{0}, \sqrt{1-\lambda^{2}} H_{0}\right)$ where $\lambda=\cos \alpha$ is applied in a direction that makes an angle $\alpha$ with the normal to the considered plate. Thus if $\lambda=1$, the imposed magnetic field is parallel to the $y$-axis and if $\lambda=0$, then the magnetic field is parallel to the plate. The magnetic Reynolds number of the flow is not taken to be small enough, the induced magnetic field is not negligible. The divergence equation $\nabla \cdot \boldsymbol{H}=0$ of Maxwell's equation for the magnetic field gives $H_{y}=\lambda H_{0}$. We use the relation $\nabla \cdot \boldsymbol{J}=0$ for the current density $\boldsymbol{J}=\left(J_{x}, J_{y}, J_{z}\right)$ where $J_{y}=$ constant . Since the plate is non conducting, $J_{y}=0$ at the plate and hence zero everywhere. Within the framework of the above stated assumptions, the equations relevant to the two-dimensional problem are governed by the following system of coupled non-linear partial differential equations.

Continuity equation

$$
\frac{\partial u}{\partial x}+\frac{\partial v}{\partial y}=0
$$

Momentum equations 


$$
\begin{aligned}
& \frac{\partial u}{\partial t}+u \frac{\partial u}{\partial x}+v \frac{\partial u}{\partial y}=v \frac{\partial^{2} u}{\partial y^{2}}+g \beta_{T}\left(T-T_{\infty}\right)+g \beta_{C}\left(C-C_{\infty}\right)-\frac{\mu}{\rho k_{l}} u+\frac{\mu_{e} \lambda H_{0}}{4 \pi \rho} \frac{\partial H_{x}}{\partial y} \\
& \frac{\partial w}{\partial t}+u \frac{\partial w}{\partial x}+v \frac{\partial w}{\partial y}=v \frac{\partial^{2} w}{\partial y^{2}}-\frac{\mu}{\rho k_{l}} w+\frac{\mu_{e} \lambda H_{0}}{4 \pi \rho} \frac{\partial H_{z}}{\partial y}
\end{aligned}
$$

Magnetic induction equations

$$
\begin{aligned}
& \frac{\partial H_{x}}{\partial t}+v \frac{\partial H_{x}}{\partial y}=\eta \frac{\partial^{2} H_{x}}{\partial y^{2}}+H_{x} \frac{\partial u}{\partial x}+\lambda H_{0} \frac{\partial u}{\partial y}-\lambda \beta_{e} \eta \frac{\partial^{2} H_{z}}{\partial y^{2}}, \\
& \frac{\partial H_{z}}{\partial t}+v \frac{\partial H_{z}}{\partial y}=\eta \frac{\partial^{2} H_{z}}{\partial y^{2}}+H_{x} \frac{\partial w}{\partial x}+\lambda H_{0} \frac{\partial w}{\partial y}+\lambda \beta_{e} \eta \frac{\partial^{2} H_{x}}{\partial y^{2}},
\end{aligned}
$$

Energy equation

$$
\begin{aligned}
& \frac{\partial T}{\partial t}+u \frac{\partial T}{\partial x}+v \frac{\partial T}{\partial y}=\frac{\kappa}{\rho c_{p}} \frac{\partial^{2} T}{\partial y^{2}}+\frac{D k_{t}}{c_{s} c_{p}} \frac{\partial^{2} C}{\partial y^{2}}+\frac{1}{16 \pi^{2} \rho c_{p} \sigma}\left(\left(\frac{\partial H_{z}}{\partial y}\right)^{2}+\left(\frac{\partial H_{x}}{\partial y}\right)^{2}\right)+ \\
& -\frac{1}{\rho c_{p}} \frac{\partial q_{r}}{\partial y}+\frac{Q}{\rho c_{p}}\left(T-T_{\infty}\right)^{p} .
\end{aligned}
$$

Concentration equation

$$
\frac{\partial C}{\partial t}+u \frac{\partial C}{\partial x}+v \frac{\partial C}{\partial y}=D \frac{\partial^{2} C}{\partial y^{2}}+\frac{D k_{t}}{T_{m}} \frac{\partial^{2} T}{\partial y^{2}}-k_{0}\left(C-C_{\infty}\right)^{q} .
$$

In addition, the boundary conditions for the model are

$$
\begin{aligned}
& u=U_{\infty}, \quad v=0, \quad w=0, \quad H_{x}=H_{w}, \quad H_{z}=0, \quad T=T_{w}, \quad C=C_{w} \quad \text { at } \quad y=0, \\
& u=0, \quad v=0, \quad w=0, \quad H_{x}=0, \quad H_{z}=0, \quad T \rightarrow 0, \quad C \rightarrow 0 \quad \text { as } \quad y \rightarrow \infty
\end{aligned}
$$

where $u, v$ and $w$ are the $x, y$ and $z$ components of the velocity vector, $H_{x}$ and $H_{z}$ are the $x$ and $z$ components of the magnetic induction vector, $H_{0}$ is the uniform magnetic field, $H_{w}$ is the induced magnetic field at the wall, $g$ is the local acceleration due to gravity, $\beta_{T}$ is the thermal expansion coefficient; $\beta_{C}$ is the concentration expansion coefficient, $v$ is the kinematics coefficient of viscosity, $\mu$ is the fluid viscosity, $\mu_{e}$ is the magnetic permeability, $\rho$ is the density of the fluid, $\kappa$ is the thermal conductivity, $c_{p}$ is the specific heat at the constant pressure, $k_{0}$ is the rate of chemical reaction and $D$ is the coefficient of mass diffusivity, $k_{t}$ is the thermal diffusion ratio, $c_{s}$ is the concentration susceptibility, respectively. Here $p$ and $q$ are considered as positive constant. The radiative heat flux $q_{r}$ is described by the Rosseland approximation (Brewster [14]) such that $q_{r}=-\frac{4 \sigma^{*}}{3 k^{*}} \frac{\partial T^{4}}{\partial y}$, where $\sigma^{*}$ and $k^{*}$ are the StefanBoltzman constant and the mean absorption coefficient, respectively. If the temperature difference within the flow is sufficiently small so that $T^{4}$ can be expressed as a linear function after using Taylor series to expand 
$T^{4}$ about the free stream temperature $T_{\infty}$ and neglecting higher-order terms, Then we have the following approximation: $T^{4} \approx 4 T_{\infty}^{3} T-3 T_{\infty}^{4}$.

\section{Mathematical formulation}

Since the solutions of the governing Eqs (2.1)-(2.7) under the boundary conditions (2.8) will be based on the finite difference method it is required to make the said equations dimensionless. For this purpose, the following dimensionless quantities are introduced

$$
\begin{gathered}
X=\frac{x U_{\infty}}{v}, \quad Y=\frac{y U_{\infty}}{v}, \quad U=\frac{u}{U_{\infty}}, \quad V=\frac{v}{U_{\infty}}, \quad W=\frac{w}{U_{\infty}}, \quad \tau=\frac{t U_{\infty}^{2}}{v}, \\
H_{1}=\sqrt{\frac{\mu_{e}}{\rho}} \frac{H_{x}}{U_{\infty}}, \quad H_{3}=\sqrt{\frac{\mu_{e}}{\rho}} \frac{H_{z}}{U_{\infty}}, \quad \bar{T}=\frac{T-T_{\infty}}{T_{w}-T_{\infty}} \quad \text { and } \quad \bar{C}=\frac{C-C_{\infty}}{C_{w}-C_{\infty}} .
\end{gathered}
$$

Using these relations, in Eqs (2.1)-(2.7) and corresponding boundary conditions (2.8) after simplification, the following nonlinear-coupled partial differential equations in terms of dimensionless variables are obtained

$$
\begin{aligned}
& \frac{\partial U}{\partial x}+\frac{\partial V}{\partial y}=0, \\
& \frac{\partial U}{\partial \tau}+U \frac{\partial U}{\partial X}+V \frac{\partial U}{\partial Y}=\frac{\partial^{2} U}{\partial Y^{2}}+\mathrm{Gr} \bar{T}+\mathrm{Gm} \bar{C}-K U+\lambda M \frac{\partial H_{1}}{\partial Y}, \\
& \frac{\partial W}{\partial \tau}+U \frac{\partial W}{\partial X}+V \frac{\partial W}{\partial Y}=\frac{\partial^{2} W}{\partial Y^{2}}-K W+\lambda M \frac{\partial H_{3}}{\partial Y} \\
& \frac{\partial H_{1}}{\partial \tau}+V \frac{\partial H_{1}}{\partial Y}=\frac{1}{P_{m}} \frac{\partial^{2} H_{1}}{\partial Y^{2}}+H_{1} \frac{\partial U}{\partial X}+\lambda M \frac{\partial U}{\partial Y}-\frac{\lambda \beta_{e}}{P_{m}} \frac{\partial^{2} H_{3}}{\partial Y^{2}}, \\
& \frac{\partial H_{3}}{\partial \tau}+V \frac{\partial H_{3}}{\partial Y}=\frac{1}{P_{m}} \frac{\partial^{2} H_{3}}{\partial Y^{2}}+H_{1} \frac{\partial W}{\partial X}+\lambda M \frac{\partial W}{\partial Y}+\frac{\lambda \beta_{e}}{P_{m}} \frac{\partial^{2} H_{1}}{\partial Y^{2}}, \\
& \frac{\partial \bar{T}}{\partial \tau}+U \frac{\partial \bar{T}}{\partial X}+V \frac{\partial \bar{T}}{\partial Y}=\left(\frac{1+R}{\operatorname{Pr}}\right) \frac{\partial^{2} \bar{T}}{\partial y^{2}}+\mathrm{Du}^{2} \frac{\partial^{2} \bar{C}}{\partial Y^{2}}+\frac{\mathrm{Ec}}{P_{m}}\left(\left(\frac{\partial H_{3}}{\partial Y}\right)^{2}+\left(\frac{\partial H_{1}}{\partial Y}\right)^{2}\right)+\beta \bar{T}^{p}, \\
& \frac{\partial \bar{C}}{\partial \tau}+U \frac{\partial \bar{C}}{\partial X}+V \frac{\partial \bar{C}}{\partial Y}=\frac{1}{\mathrm{Sc}} \frac{\partial^{2} \bar{C}}{\partial Y^{2}}+\operatorname{Sr} \frac{\partial^{2} T}{\partial Y^{2}}-\gamma \bar{C}^{q}
\end{aligned}
$$

The boundary conditions are

$$
\begin{aligned}
& U=1, \quad V=0, \quad W=0, \quad H_{1}=\sqrt{\frac{\mu_{e}}{\rho}} \frac{H_{w}}{U_{\infty}}=1 \text { (say), } H_{3}=0, \quad \bar{T}=1, \quad \bar{C}=1, \quad \text { at } Y=0, \\
& U=0, \quad V=0, \quad W=0, \quad H_{1}=0, \quad H_{3}=0, \quad \bar{T} \rightarrow 0, \quad \bar{C} \rightarrow 0 \quad \text { as } \quad Y \rightarrow \infty
\end{aligned}
$$


where $\tau$ represents the dimensionless time, $X$ and $Y$ are the dimensionless Cartesian coordinates, $U, V$ and $W$ are the dimensionless velocity components, $H_{1}$ and $H_{3}$ are the dimensionless primary and secondary induced magnetic field, $\bar{T}$ is the dimensionless temperature, $\bar{C}$ is the dimensionless concentration, $\quad \mathrm{Gr}=\frac{g B_{T}\left(T_{w}-T_{\infty}\right) \cup}{U_{\infty}^{3}} \quad$ (Grashof number), $\mathrm{Gm}=\frac{g B_{C}\left(C_{w}-C_{\infty}\right) \cup}{U_{\infty}^{3}} \quad$ (modified Grashof number), $K=\frac{\mu v}{\rho k_{l} U_{\infty}^{2}}$ (permeability of the porous medium), $M=\frac{1}{4 \pi} \frac{H_{0}}{U_{\infty}} \sqrt{\frac{\mu_{e}}{\rho}}$ (magnetic parameter), $P_{m}=4 \pi \sigma u \mu_{e}$ (magnetic diffusivity number), $R=\frac{16 \sigma^{*} T_{\infty}^{3}}{3 k^{*} \kappa}$ (radiation parameter), $\operatorname{Pr}=\frac{\rho c_{p} \mathrm{v}}{\kappa} \quad$ (Prandtl number), $\quad \mathrm{Du}=\frac{D k_{t}}{v c_{s} c_{p}} \frac{\left(C_{w}-C_{\infty}\right)}{\left(T_{w}-T_{\infty}\right)} \quad$ (Dufour number), $\quad \mathrm{Ec}=\frac{U_{\infty}^{2}}{4 \pi c_{p}\left(T_{w}-T_{\infty}\right)} \quad$ (Eckert number), $\beta=\frac{Q v\left(T_{w}-T_{\infty}\right)^{p-1}}{\rho c_{p} U_{\infty}^{2}} \quad$ (heat generation or absorption parameter), $\mathrm{Sc}=\frac{\mathrm{v}}{D} \quad$ (Schmidt number), $\mathrm{Sr}=\frac{D k_{T}}{\mathrm{v} T_{m}} \frac{\left(T_{w}-T_{\infty}\right)}{\left(C_{w}-C_{\infty}\right)}$ (Soret number) and $\gamma=\frac{k_{0} \mathrm{v}\left(C_{w}-C_{\infty}\right)^{q-1}}{U_{\infty}^{2}}$ (chemical reaction parameter).

\section{Shear stress, current density, Nusselt and Sherwood number}

The quantities of chief physical interest are shear stress, current density, Nusselt number and Sherwood number. The following equations represent the local and average shear stress at the plate. Local shear stress in the $x$-direction, $\tau_{x L}=\mu_{0}\left(\frac{\partial u}{\partial y}\right)_{y=0}$ and average shear stress in the $x$-direction, $\tau_{x A}=\mu_{0} \int\left(\frac{\partial u}{\partial y}\right)_{y=0} d x$ which are proportional to $\left(\frac{\partial U}{\partial Y}\right)_{Y=0}$ and $\int_{0}^{100}\left(\frac{\partial U}{\partial Y}\right)_{Y=0} d X$ are given respectively. The local shear stress in the $z$ direction, $\tau_{z L}=\mu_{0}\left(\frac{\partial w}{\partial y}\right)_{y=0}$ and average shear stress in $z$-direction, $\tau_{z A}=\mu_{0} \int\left(\frac{\partial w}{\partial y}\right)_{y=0} d x$ which are proportional to $\left(\frac{\partial W}{\partial Y}\right)_{Y=0}$ and $\int_{0}^{100}\left(\frac{\partial W}{\partial Y}\right)_{Y=0} d X$ are given respectively. From the induced magnetic field, the effects of various parameters on the local and average current density have been investigated. The following equations represent the local and average current density at the plate: local current density in the $x$-direction, $j_{x L}=\mu_{0}\left(-\frac{\partial H_{x}}{\partial y}\right)_{y=0}$ and average current density in $x$-direction, $j_{x A}=\mu_{0} \int\left(-\frac{\partial H_{x}}{\partial y}\right)_{y=0} d x$ which are proportional to $\left(-\frac{\partial H_{I}}{\partial Y}\right)_{Y=0}$ and $\int_{0}^{100}\left(-\frac{\partial H_{1}}{\partial Y}\right)_{Y=0} d X$, respectively. The local current density in the $z$-direction, $j_{z L}=\mu_{0}\left(-\frac{\partial H_{z}}{\partial y}\right)_{y=0}$ and average current density in the $z$-direction, $j_{z A}=\mu_{0} \int\left(-\frac{\partial H_{z}}{\partial y}\right)_{y=0} d x$ which are 
proportional to $\left(-\frac{\partial H_{3}}{\partial Y}\right)_{Y=0}$ and $\int_{0}^{100}\left(-\frac{\partial H_{3}}{\partial Y}\right)_{Y=0} d X$, respectively. From the temperature field, the effects of various parameters on the local and average heat transfer coefficients have been studied. The following equations represent the local and average heat transfer rate that is the well known Nusselt number, local Nusselt number, $\mathrm{Nu}_{\mathrm{L}}=-\mu_{0}\left(\frac{\partial T}{\partial y}\right)_{y=0}$ and average Nusselt number, $\mathrm{Nu}_{\mathrm{A}}=-\mu_{0} \int\left(\frac{\partial T}{\partial y}\right)_{y=0} d x$ which are proportional to $-\left(\frac{\partial \bar{T}}{\partial Y}\right)_{Y=0}$ and $-\int_{0}^{100}\left(-\frac{\partial \bar{T}}{\partial Y}\right)_{Y=0} d X$, respectively. And from the concentration field, the effects of various parameters on the local and average mass transfer coefficients have been analyzed. The following equations represent the local and average mass transfer rate, that is the well known Sherwood number, local Sherwood number, $\mathrm{Sh}_{\mathrm{L}}=-\mu_{0}\left(\frac{\partial C}{\partial y}\right)_{y=0}$ and average Sherwood number, $\mathrm{Sh}_{\mathrm{A}}=-\mu_{0} \int\left(\frac{\partial C}{\partial y}\right)_{y=0} d x$ which are proportional to $-\left(\frac{\partial \bar{C}}{\partial Y}\right)_{Y=0}$ and $-\int_{0}^{100}\left(-\frac{\partial \bar{C}}{\partial Y}\right)_{Y=0} d X$, respectively.

\section{Numerical solutions}

To solve the non-dimensional system by the explicit finite difference method, a set of finite difference equations. To obtain the difference equations the region of the flow is divided into a grid or mesh of lines parallel to the $X$ and $Y$ axes where the $X$-axis is taken along the plate and the $Y$-axis is normal to the plate.

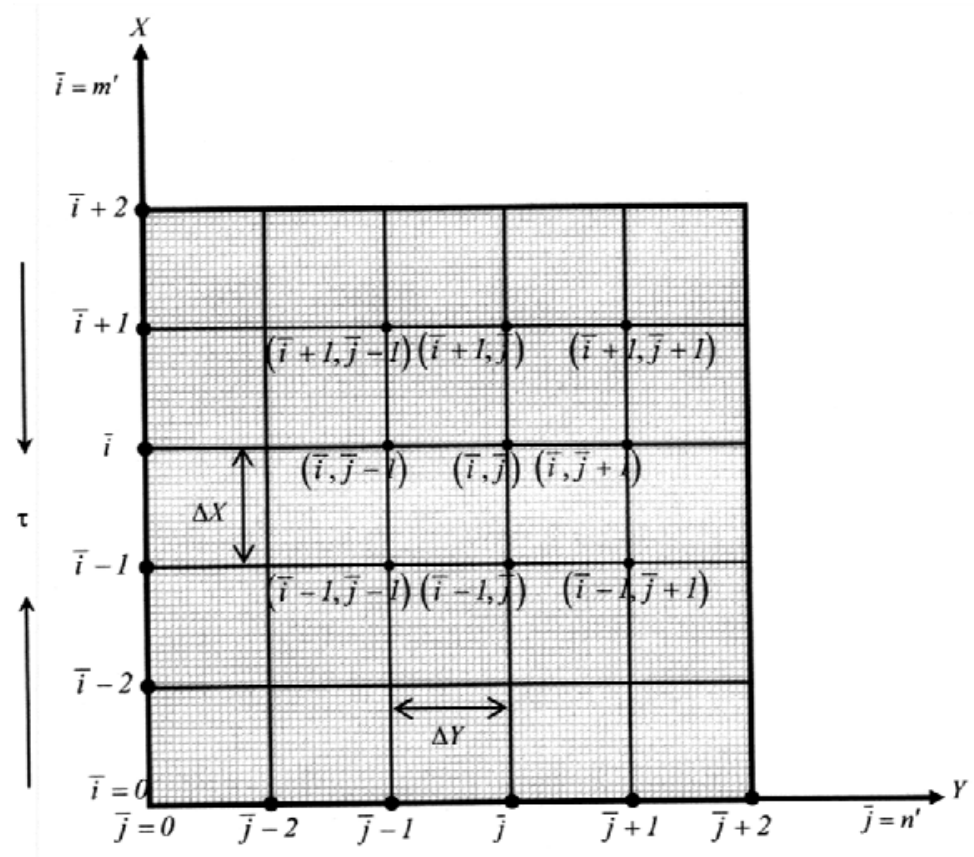

Fig.2. Explicit finite difference system grid.

Here the plate of height $X_{\max }(=100)$ is considered, i.e. $X$ varies from 0 to 100 and $Y_{\max }(=35)$ is assumed as corresponding to $Y \rightarrow \infty$, i.e. $Y$ varies from 0 to 35 . There are $m^{\prime}(=200)$ and $n^{\prime}(=200)$ 
grid spacing in the $X$ and $Y$ directions respectively as shown Fig.2. $\Delta X, \Delta Y$ are constant mesh sizes along the $X$ and $Y$ directions respectively and are taken as follows, $\Delta X=0.5(0 \leq X \leq 100)$ and $\Delta Y=0.175(0 \leq Y \leq 35)$ with the smaller time-step, $\Delta \tau=0.005$. Let $U^{\prime}, W^{\prime}, H_{1}^{\prime}, H_{3}^{\prime}, \bar{T}^{\prime}$ and $\bar{C}^{\prime}$ denote the values of $U, W, H_{1}, H_{3}, \bar{T}$ and $\bar{C}$ at the end of a time-step, respectively. Using the explicit finite difference approximation, the system of partial differential Eqs (3.1)-(3.7) and the boundary conditions (3.8), we obtain an appropriate set of finite difference equations

$$
\begin{aligned}
& \frac{U_{\bar{i}, \bar{j}}^{\prime}-U_{\bar{i}-1, \bar{j}}^{\prime}}{\Delta X}+\frac{V_{\bar{i}, \bar{j}}-V_{\bar{i}, \bar{j}-1}}{\Delta Y}=0, \\
& \frac{U_{\bar{i}, \bar{j}}^{\prime}-U_{\bar{i}, \bar{j}}}{\Delta \tau}+U_{\bar{i}, \bar{j}} \frac{U_{\bar{i}, \bar{j}}-U_{\bar{i}-1, \bar{j}}}{\Delta X}+V_{\bar{i}, \bar{j}} \frac{U_{\bar{i}, \bar{j}+1}-U_{\bar{i}, \bar{j}}}{\Delta Y}=\frac{U_{\bar{i}, \bar{j}+1}-2 U_{\bar{i}, \bar{j}}+U_{\bar{i}, \bar{j}}}{(\Delta Y)^{2}}+ \\
& +\mathrm{Gr} \bar{T}_{\bar{i}, \bar{j}}+\mathrm{Gm} \bar{C}_{\bar{i}, \bar{j}}-K U_{\bar{i}, \bar{j}}+\lambda M \frac{H_{l \bar{i}, \bar{j}-1}-H_{l \bar{i}, \bar{j}}}{\Delta Y}, \\
& \frac{W_{\bar{i}, \bar{j}}-W_{\bar{i}-1, \bar{j}}}{\Delta X}+U_{\bar{i}, \bar{j}} \frac{W_{\bar{i}, \bar{j}}-W_{\bar{i}-1, \bar{j}}}{\Delta X}+V_{\bar{i}, \bar{j}} \frac{W_{\bar{i}, \bar{j}+1}-W_{\bar{i}, \bar{j}}}{\Delta Y}=\frac{W_{\bar{i}, \bar{j}+1}-2 W_{\bar{i}, \bar{j}}+W_{\bar{i}, \bar{j}}}{(\Delta Y)^{2}}+ \\
& -K W_{\bar{i}, \bar{j}}+\lambda M \frac{H_{3 \bar{i}, \bar{j}-1}-H_{3 \bar{i}, \bar{j}}}{\Delta Y}, \\
& \frac{H_{\bar{l}, \bar{j}}^{\prime}-H_{1 \bar{i}, \bar{j}}}{\Delta \tau}+V_{\bar{i}, \bar{j}} \frac{H_{l \bar{i}, \bar{j}-1}-H_{1 \bar{i}, \bar{j}}}{\Delta Y}=\frac{1}{P_{m}} \frac{H_{1 \bar{i}, \bar{j}+1}-2 H_{1 \bar{i}, \bar{j}}+H_{1 \bar{i}, \bar{j}-1}}{(\Delta Y)^{2}}+ \\
& +H_{l \bar{i}, \bar{j}} \frac{U_{\bar{i}, \bar{j}}-U_{\bar{i}-1, \bar{j}}}{\Delta X}+\lambda M \frac{U_{\bar{i}, \bar{j}+1}-U_{\bar{i}, \bar{j}}}{\Delta Y}-\frac{\lambda \beta_{e}}{P_{m}} \frac{H_{3 \bar{i}, \bar{j}+1}-2 H_{3 \bar{i}, \bar{j}}+H_{3 \bar{i}, \bar{j}-1}}{(\Delta Y)^{2}}, \\
& \frac{H_{\overline{3}, \bar{j}}^{\prime}-H_{3 \bar{i}, \bar{j}}}{\Delta \tau}+V_{\bar{i}, \bar{j}} \frac{H_{3 \bar{i}, \bar{j}-1}-H_{3 \bar{i}, \bar{j}}}{\Delta Y}=\frac{1}{P_{m}} \frac{H_{3 \bar{i}, \bar{j}+1}-2 H_{3 \bar{i}, \bar{j}}+H_{3 \bar{i}, \bar{j}-1}}{(\Delta Y)^{2}}+ \\
& +H_{l \bar{i}, \bar{j}} \frac{W_{\bar{i}, \bar{j}}-W_{\bar{i}-1, \bar{j}}}{\Delta X}+\lambda M \frac{W_{\bar{i}, \bar{j}+1}-W_{\bar{i}, \bar{j}}}{\Delta Y}+\frac{\lambda \beta_{e}}{P_{m}} \frac{H_{l \bar{i}, \bar{j}+1}-2 H_{1 \bar{l}, \bar{j}}+H_{1 \bar{i}, \bar{j}-1}}{(\Delta Y)^{2}}, \\
& \frac{\bar{T}_{\bar{i}, \bar{j}}^{\prime}-\bar{T}_{\bar{i}, \bar{j}}}{\Delta \tau}+U_{\bar{i}, \bar{j}} \frac{\bar{T}_{\bar{i}, \bar{j}}-\bar{T}_{\bar{i}-1, \bar{j}}}{\Delta X}+V_{\bar{i}, \bar{j}} \frac{\bar{T}_{\bar{i}, \bar{j}+1}-\bar{T}_{\bar{i}, \bar{j}}}{\Delta Y}=\left(\frac{1+R}{\operatorname{Pr}}\right) \frac{\bar{T}_{\bar{i}, \bar{j}+1}-2 \bar{T}_{\bar{i}, \bar{j}}+\bar{T}_{\bar{i}, \bar{j}-1}}{(\Delta Y)^{2}}+ \\
& +\mathrm{Du} \frac{\bar{C}_{\bar{i}, \bar{j}+1}-2 \bar{C}_{\bar{i}, \bar{j}}+\bar{C}_{\bar{i}, \bar{j}-1}}{(\Delta Y)^{2}}+\frac{\operatorname{Ec}}{P_{m}}\left(\left(\frac{H_{3 \bar{i}, \bar{j}-1}-H_{3 \bar{i}, \bar{j}}}{\Delta Y}\right)^{2}+\left(\frac{H_{l \bar{i}, \bar{j}-1}-H_{l \bar{i}, \bar{j}}}{\Delta Y}\right)^{2}\right)+ \\
& +\beta\left(\bar{T}_{i, j}\right)^{p},
\end{aligned}
$$




$$
\begin{aligned}
& \frac{\bar{C}_{\bar{i}, \bar{j}}^{\prime}-\bar{C}_{\bar{i}, \bar{j}}}{\Delta \tau}+U_{\bar{i}, \bar{j}} \frac{\bar{C}_{\bar{i}, \bar{j}}-\bar{C}_{\bar{i}-1, \bar{j}}}{\Delta X}+V_{\bar{i}, \bar{j}} \frac{\bar{C}_{\bar{i}, \bar{j}+1}-\bar{C}_{\bar{i}, \bar{j}}}{\Delta Y}=\frac{1}{\mathrm{Sc}} \frac{\bar{C}_{\bar{i}, \bar{j}+1}-2 \bar{C}_{\bar{i}, \bar{j}}+\bar{C}_{\bar{i}, \bar{j}-1}}{(\Delta Y)^{2}}+ \\
& +\operatorname{Sr} \frac{\bar{T}_{\bar{i}, \bar{j}+1}-2 \bar{T}_{\bar{i}, \bar{j}}+\bar{T}_{\bar{i}, \bar{j}-1}}{(\Delta Y)^{2}}-\gamma\left(\bar{C}_{\bar{i}, \bar{j}}\right)^{q},
\end{aligned}
$$

with the boundary conditions

$$
\begin{aligned}
& U_{\bar{i}, 0}^{n}=1, \quad W_{\bar{i}, 0}^{n}=0, \quad H_{1 \bar{i}, 0}^{n}=1, \quad H_{3 \bar{i}, 0}^{n}=0, \quad \bar{T}_{\bar{i}, 0}^{n}=1, \quad \bar{C}_{\bar{i}, 0}^{n}=1, \\
& U_{\bar{i}, L}^{n}=0, \quad W_{\bar{i}, L}^{n}=0, \quad H_{1 \bar{i}, L}^{n}=0, \quad H_{3 \bar{i}, L}^{n}=0, \quad \bar{T}_{\bar{i}, L}^{n}=0, \quad \bar{C}_{\bar{i}, L}^{n}=0 \quad \text { where } L \rightarrow \infty .
\end{aligned}
$$

Here the subscripts $\bar{i}$ and $\bar{j}$ designate the grid points with $X$ and $Y$ coordinates respectively and the superscript $n$ represents a value of time, $\tau=n \Delta \tau$ where $n=0,1,2, \ldots \ldots \ldots$. The primary velocity $(U)$, secondary velocity $(W)$, primary induced magnetic field $\left(H_{1}\right)$, secondary induced magnetic field $\left(H_{3}\right)$, temperature $(\bar{T})$ and concentration $(\bar{C})$ distributions at all interior nodal points may be computed by successive applications of the above finite difference equations. The numerical values of the local shear stresses, local Nusselt number and local Sherwood number are evaluated by five-point approximate formula for the derivatives and then the average shear stress, current density, Nusselt number and Sherwood number are calculated by the use of Simpson's $\frac{1}{3}$ integration formula. The obtained values are graphically shown in Figs 3-38. The stability conditions of the explicit finite difference method are $K \frac{\Delta \tau}{2}+U \frac{\Delta \tau}{\Delta X}+|-V| \frac{\Delta \tau}{\Delta Y}+\frac{2 \Delta \tau}{(\Delta Y)^{2}} \leq 1, \quad|-V| \frac{\Delta \tau}{\Delta Y}+\frac{1}{P_{m}} \frac{2 \Delta \tau}{(\Delta Y)^{2}} \leq 1$, $U \frac{\Delta \tau}{\Delta X}+|-V| \frac{\Delta \tau}{\Delta Y}+\left(\frac{1+R}{\operatorname{Pr}}\right) \frac{2 \Delta \tau}{(\Delta Y)^{2}}-\beta \bar{T}^{p-1} \leq 1$ and $U \frac{\Delta \tau}{\Delta X}+|-V| \frac{\Delta \tau}{\Delta Y}+\frac{1}{\operatorname{Sc}} \frac{2 \Delta \tau}{(\Delta Y)^{2}}+\gamma \bar{C}^{q-1} \leq 1$. When $\Delta \tau, \Delta X$ and $\Delta Y$ approach zero, then the problem will be converged. That means the results of the explicit finite difference method approach the true solutions.

\section{Results and discussion}

To investigate the physical situation of the problem, the numerical values and graphs of nondimensional primary velocity $(U)$, secondary velocity $(W)$, primary induced magnetic field $\left(H_{1}\right)$, secondary induced magnetic field $\left(H_{3}\right)$, temperature $(\bar{T})$ and concentration $(\bar{C})$ distributions within the boundary layer have been computed for different values of permeability of the porous medium $(K),(\alpha)$, magnetic parameter $(M)$, magnetic diffusivity number $\left(P_{m}\right)$, Hall parameter $\left(\beta_{e}\right)$, radiation parameter $(R)$, Prandtl number $(\mathrm{Pr})$, Dufour number $(\mathrm{Du})$, Eckert number $(\mathrm{Ec})$, heat generation or absorption parameter $(\beta)$, Schmidt number $(\mathrm{Sc})$, Soret number $(\mathrm{Sr})$ and chemical reaction parameter $(\gamma)$ with the help of a computer programming language Compaq Visual Fortran 6.6a and Tecplot 7. These computed numerical results have been shown graphically. To obtain the steady-state solutions, the computation has been carried out up to $\tau=80$. It is observed that the numerical values of $U, W, H_{1}, H_{2}, \bar{T}$ and $\bar{C}$ show little changes after $\tau=60$. Hence at $\tau=60$, the solutions of all variables are steady-state solutions. 
To observe the physical situation of the problem, the steady-state solutions have been illustrated in Figs 3-38 when $p=2$ and $q=2$. The primary velocity profiles have been displayed for various values of the permeability of the porous medium $(K),(\alpha)$ and magnetic parameter $(M)$ respectively illustrated in Figs 3-4a. These results show that the fluid primary velocity decreases with the increase of permeability of the porous medium. It has been observed that the primary velocity of the fluid increases within the interval $0<Y<6$ (approx.), then decreases with the increase of $(\alpha)$. For the effects the magnetic parameter, it has been observed that the primary velocity of the fluid decreases within the interval $0<Y<6$ (approx.) with the increase of the magnetic parameter. While reverse effects are found with the increase of the magnetic parameter. Figures $4 \mathrm{~b}-5$ show the secondary velocity profiles for various values of the permeability of the porous medium $(K),(\alpha)$ and magnetic parameter $(M)$, respectively. It has been observed that the secondary velocity of the fluid decreases within the interval $0<Y<4$ (approx.), then increases with the increase of permeability of the porous medium and $(\alpha)$ while opposite effects are found with the increase of the magnetic parameter. The primary induced magnetic fields have been displayed for various values of the permeability of the porous medium $(K),(\alpha)$, magnetic parameter $(M)$, magnetic diffusivity number $\left(P_{m}\right)$ and Hall parameter $\left(\beta_{e}\right)$ respectively, in Figs 6-8a. These results show that the fluid primary induced magnetic field increases with the increase of the permeability of the porous medium and $(\alpha)$ while decreases with the increase of the magnetic parameter. For the effects of the magnetic diffusivity number, it has been seen that the primary induced magnetic field decreases within the interval $0<Y<16$ (approx.), then increases for $Y>16$. It has been also seen that the primary induced magnetic field increases within the interval $0<Y<11$ (approx.), and decreases for $Y>16$ with the increase of the Hall parameter. Figures 8b-10 display the secondary induced magnetic fields for various values of the permeability of the porous medium $(K),(\alpha)$, magnetic parameter $(M)$, magnetic diffusivity number $\left(P_{m}\right)$ and Hall parameter $\left(\beta_{e}\right)$ respectively. These results show that the fluid secondary induced magnetic field decreases within the interval $0<Y<16$ (approx.) with the increase of the permeability of the porous medium and $(\alpha)$ while increases for $Y>16$. For the effects of the magnetic diffusivity number, the fluid secondary induced magnetic field increases within the interval $0<Y<6$ (approx.) while opposite effects have been found for $Y>6$. Figures 11-13a display the fluid temperature distributions for various values of the magnetic diffusivity number $\left(P_{m}\right)$, radiation parameter $(R)$, Prandtl number $(\operatorname{Pr})$ and heat generation or absorption parameter $(\beta)$, Dufour number $(\mathrm{Du})$ and Eckert number $(\mathrm{Ec})$, respectively. These results show that the temperature of the fluid increases with the increase of the radiation parameter, heat generation or absorption parameter, Dufour number and Eckert number, while it decreases with the increase of the Prandtl number. It is noted that $\beta<0$ and $\beta>0$ are treated as heat absorption and genaration, respectively. As for the effects of the magnetic diffusivity number, it is observed from Fig.12a that there is no change of temperature. Figures $13 \mathrm{~b}-14$ show the fluid concentration for several values of the Prandtl number $(\mathrm{Pr})$ and heat generation or absorption parameter $(\beta)$, chemical reaction parameter $(\gamma)$ with two values of the Schmidt number $\mathrm{Sc}=0.60$ (water vapor) and $\mathrm{Sc}=0.94$ (carbon dioxide) and Soret number (Sr) respectively. The concentration slightly increases within the interval $0<Y<4$ (approx.), while opposite effects have been observed for $Y>4$. But there is no change of concentration with the increase of the heat generation or absorption parameter. It is noted that the concentration decreases with the increase of the chemical reaction parameter $(\gamma)$, where $\gamma<0$ and $\gamma>0$ are treated as generative and destructive chemical reaction, respectively. The concentration also decreases with the increase of the Schmidt number and leads to thining of the concentration boundary layers. For the effects of the Soret 
number, it has been observed that the concentration of the fluid increases with the increase of the Soret number.

(a)

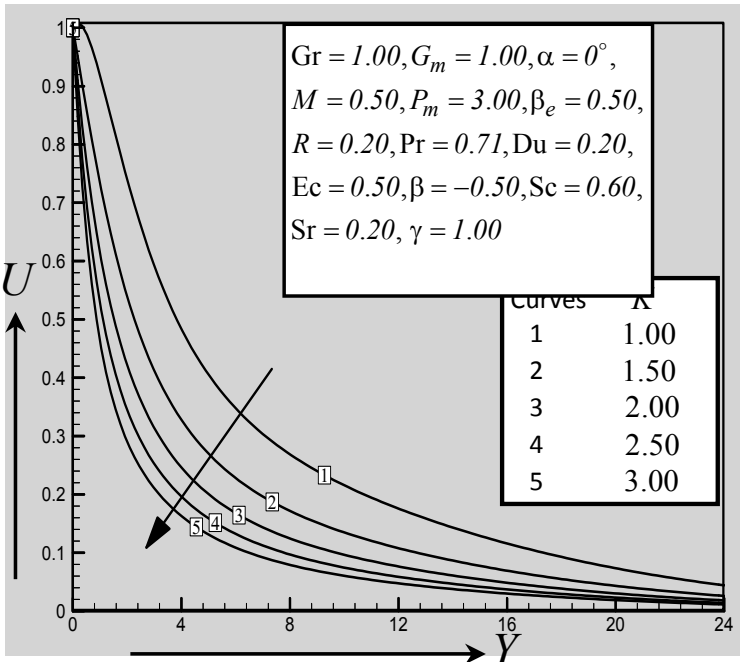

(b)

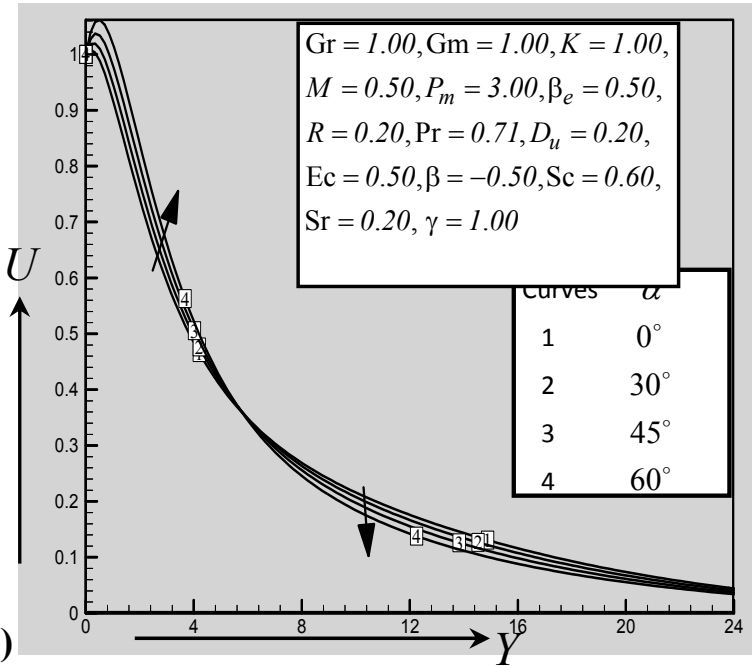

Fig.3. Illustration of primary velocity profiles for various values of (a) permeability of the porous medium $(K)$ and (b) $(\alpha)$ when $p=2$ and $q=2$.

(a)

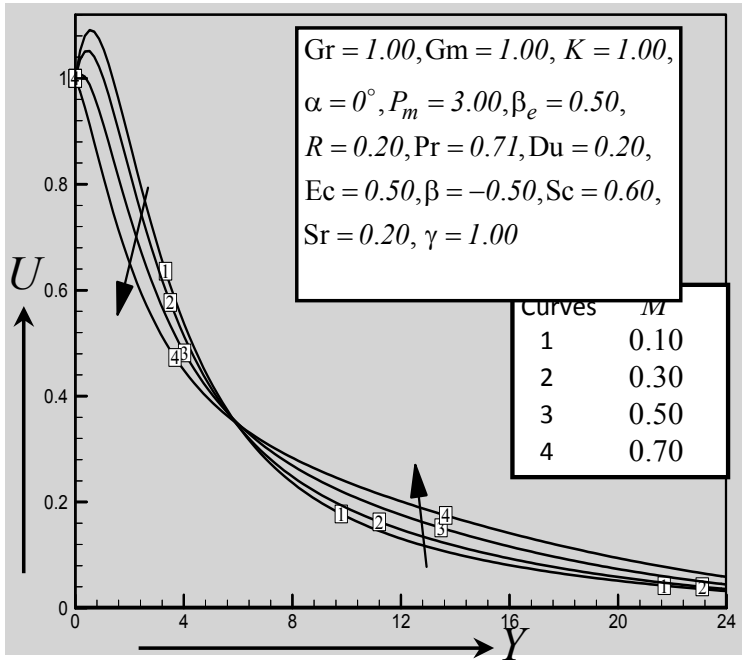

(b)

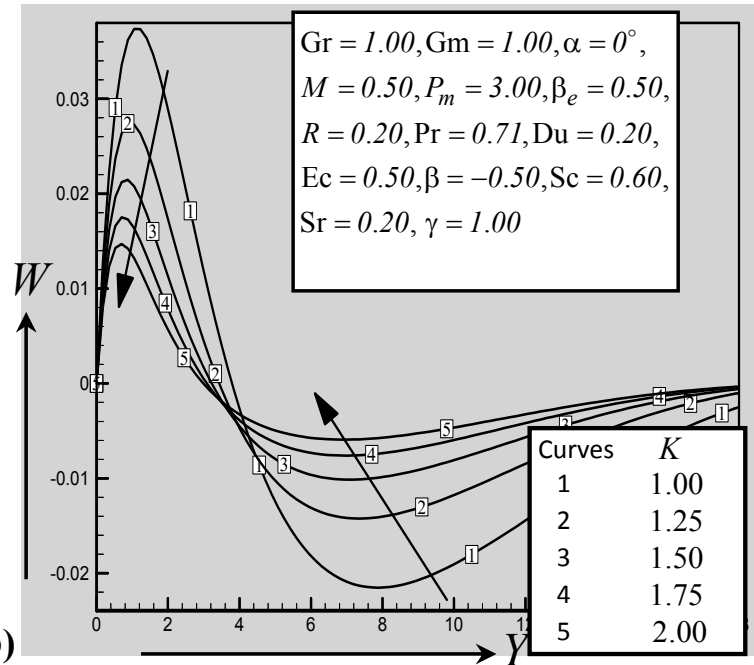

Fig.4. Illustration of (a) primary velocity profiles for various values of the magnetic parameter $(M)$ and (b) secondary velocity profiles for various values of the permeability of the porous medium $(K)$ when $p=2$ and $q=2$. 

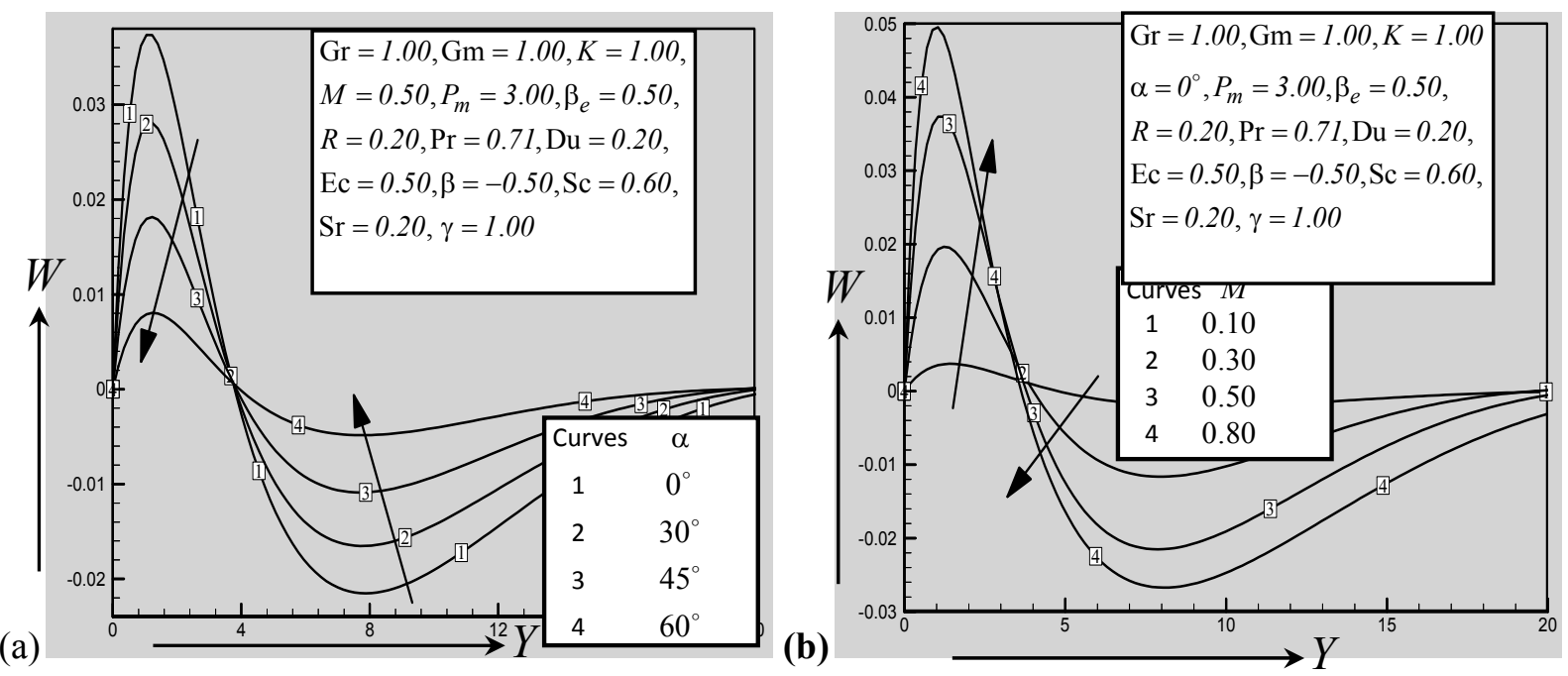

Fig.5. Illustration of secondary velocity profiles for various values of (a) $(\alpha)$ and (b) magnetic parameter $(M)$ when $p=2$ and $q=2$.
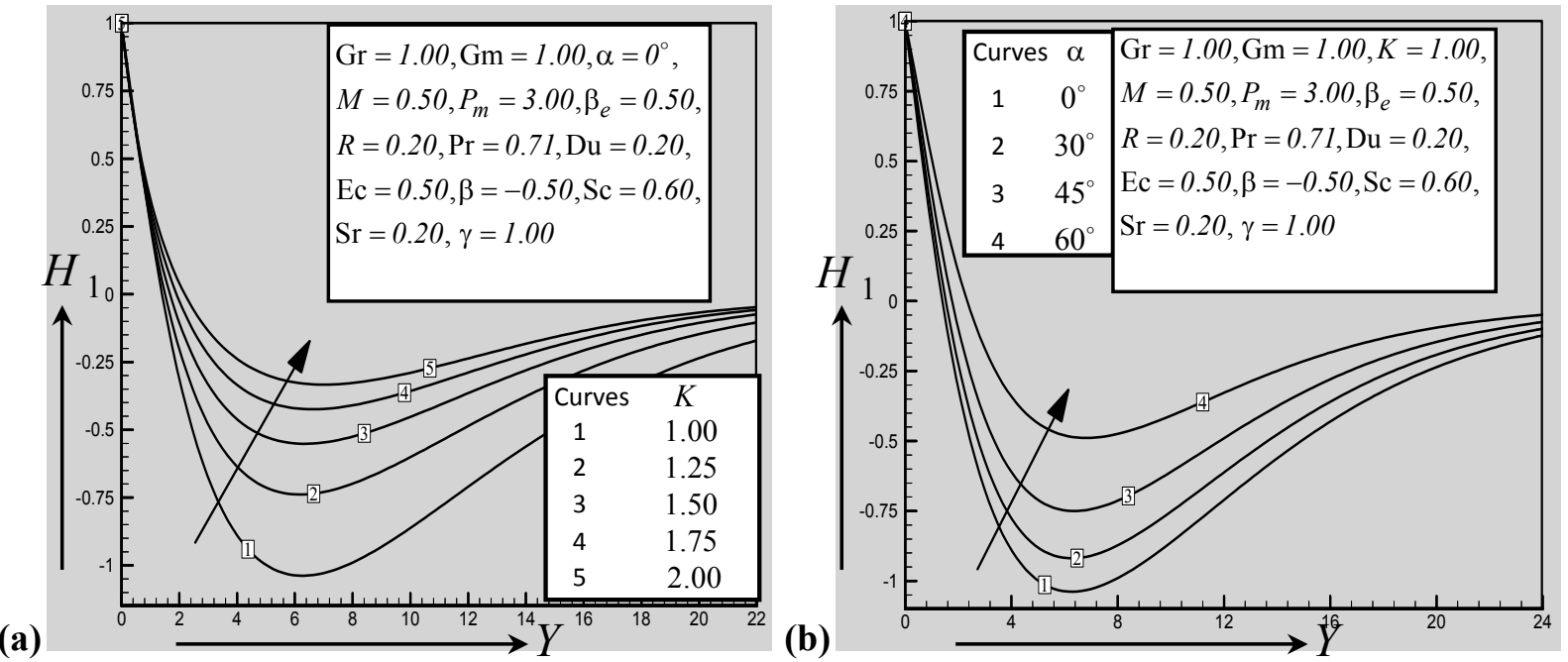

Fig.6. Illustration of primary induced magnetic field profiles for various values of (a) permeability of the porous medium $(K)$ and (b) $(\alpha)$ when $p=2$ and $q=2$. 
(a)

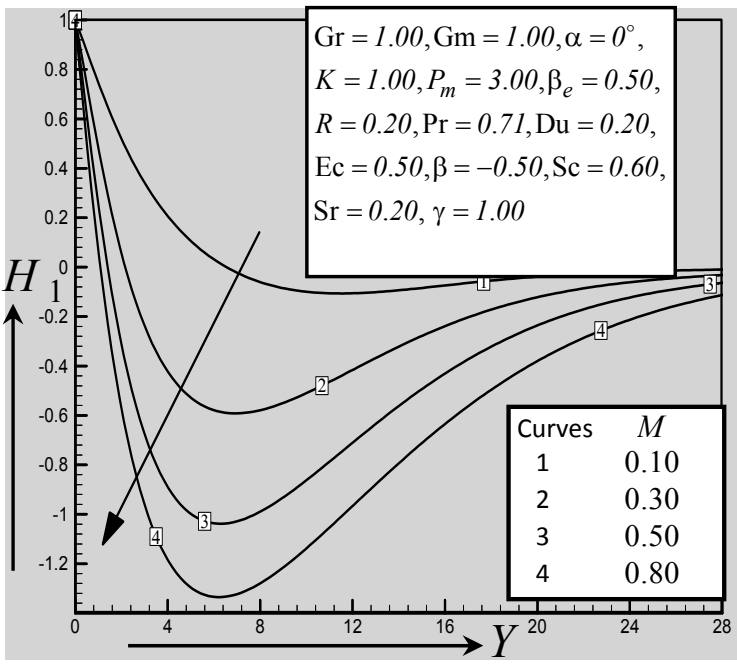

(b)

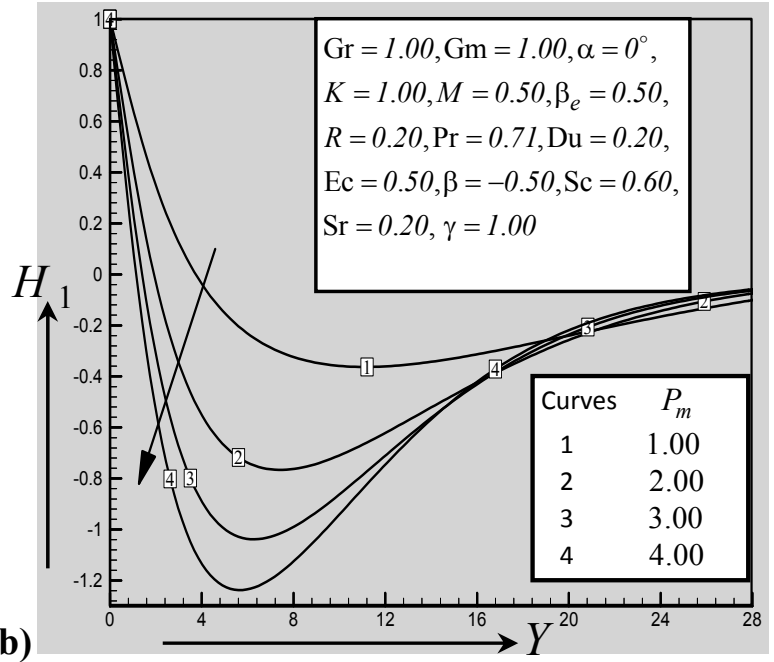

Fig.7. Illustration of primary induced magnetic field profiles for various values of (a) magnetic parameter $(M)$ and (b) magnetic diffusivity number $\left(P_{m}\right)$ when $p=2$ and $q=2$.
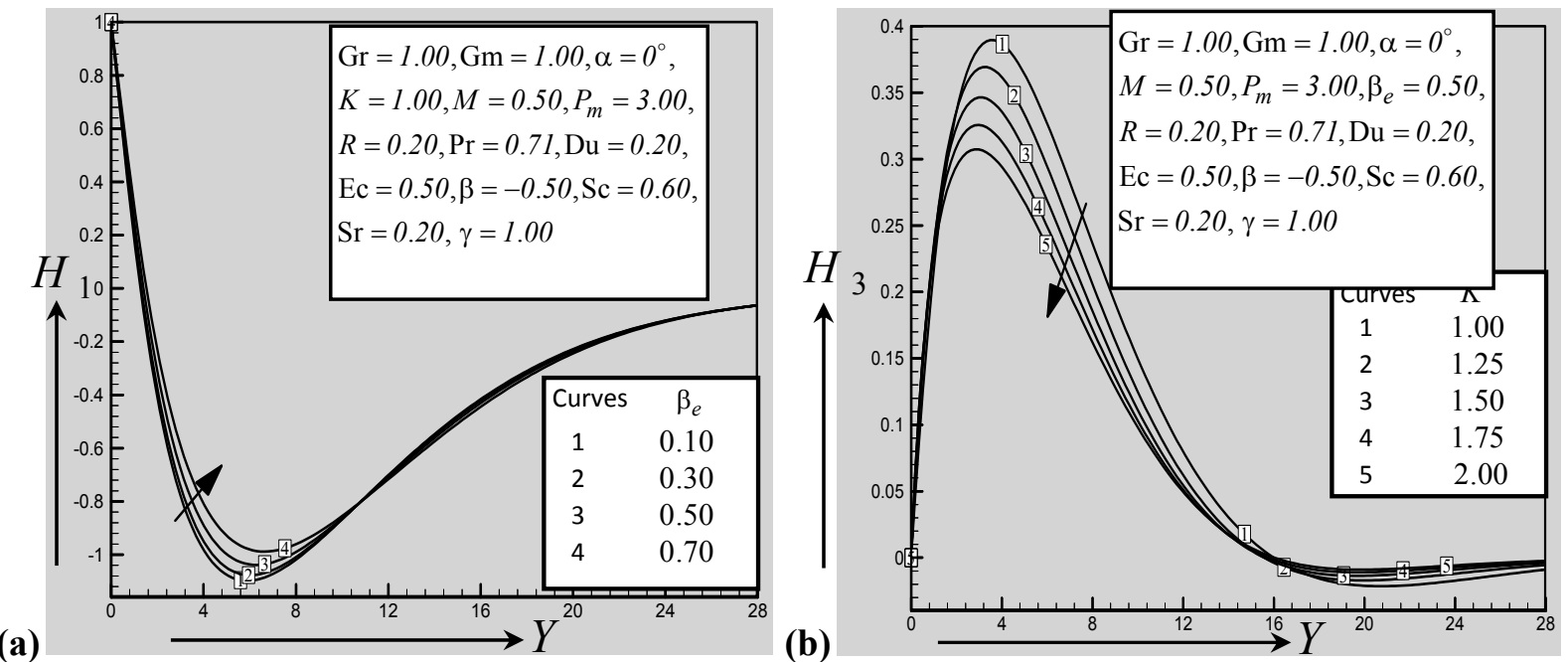

Fig.8. Illustration of (a) primary induced magnetic field profiles for various values of the Hall current parameter $\left(\beta_{e}\right)$ and $(b)$ secondary induced magnetic field profiles for various values of the permeability of the porous medium $(K)$ when $p=2$ and $q=2$. 

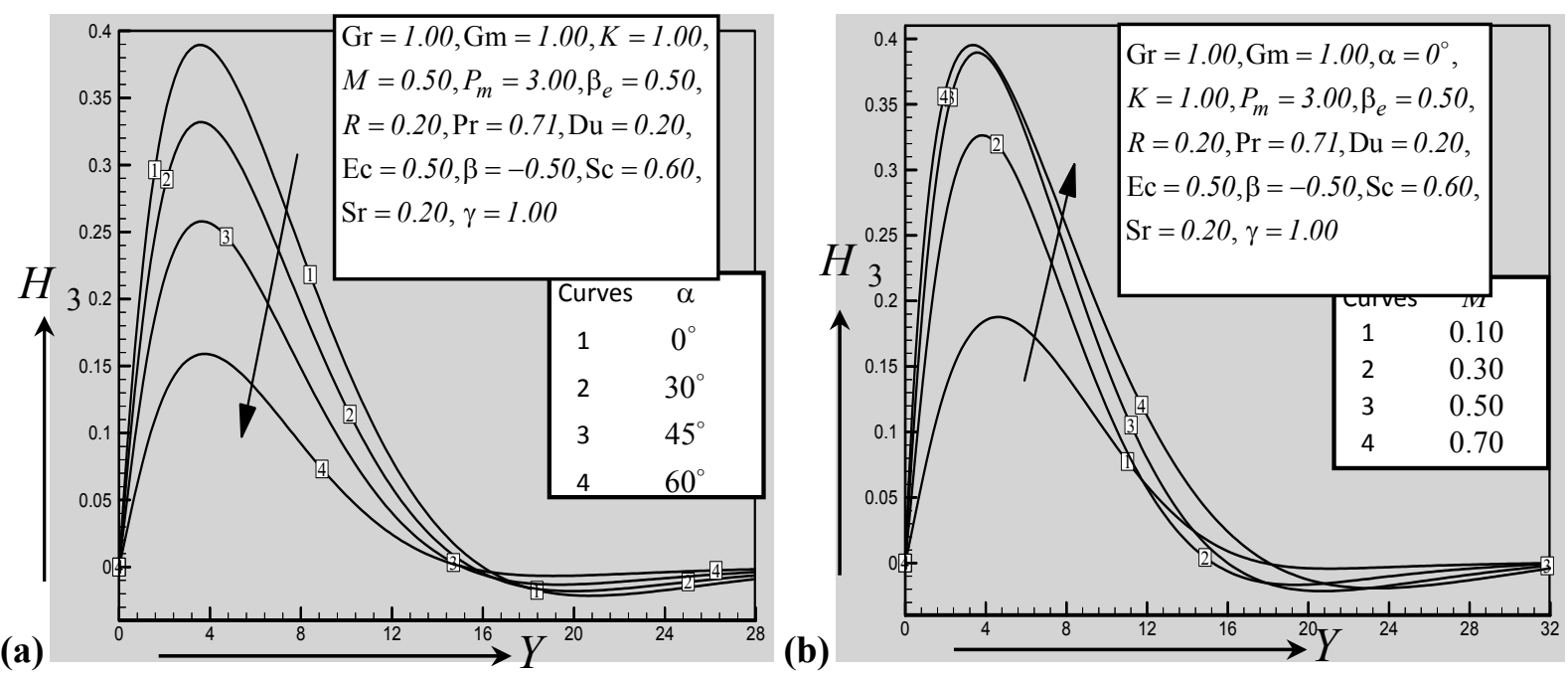

Fig.9. Illustration of secondary induced magnetic field profiles for various values of (a) ( $\alpha$ ) and (b) magnetic parameter $(M)$ when $p=2$ and $q=2$.
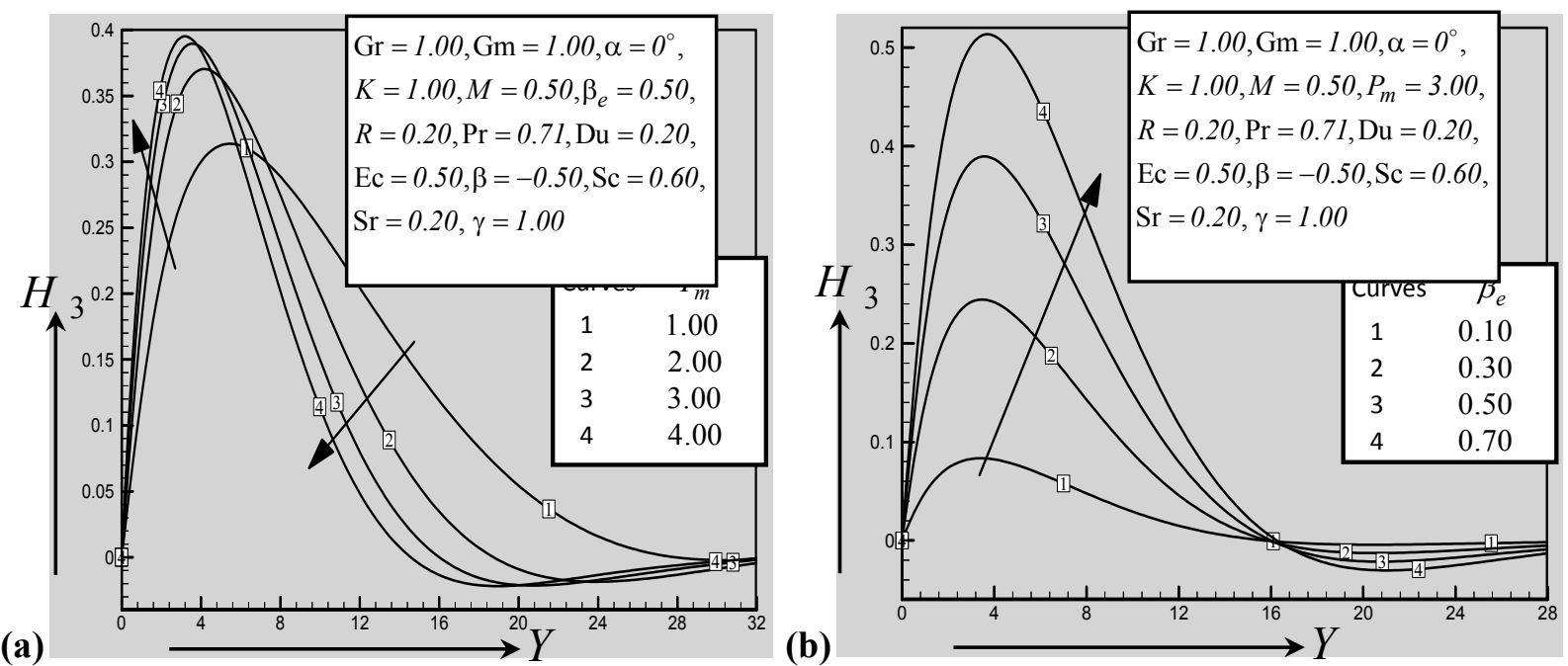

Fig.10. Illustration of secondary magnetic induced field profiles for various values of (a) magnetic diffusivity number $\left(P_{m}\right)$ and (b) Hall current parameter $\left(\beta_{e}\right)$ when $p=2$ and $q=2$. 
(a)

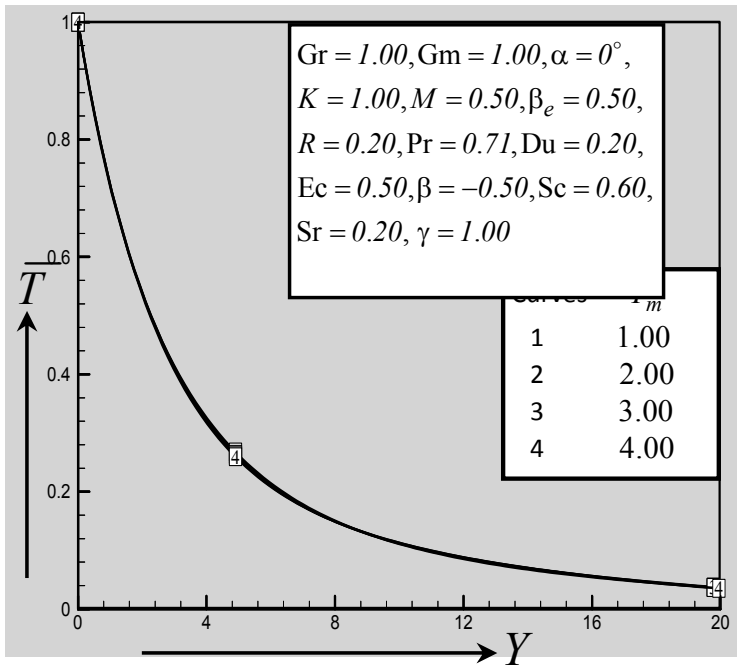

(b)

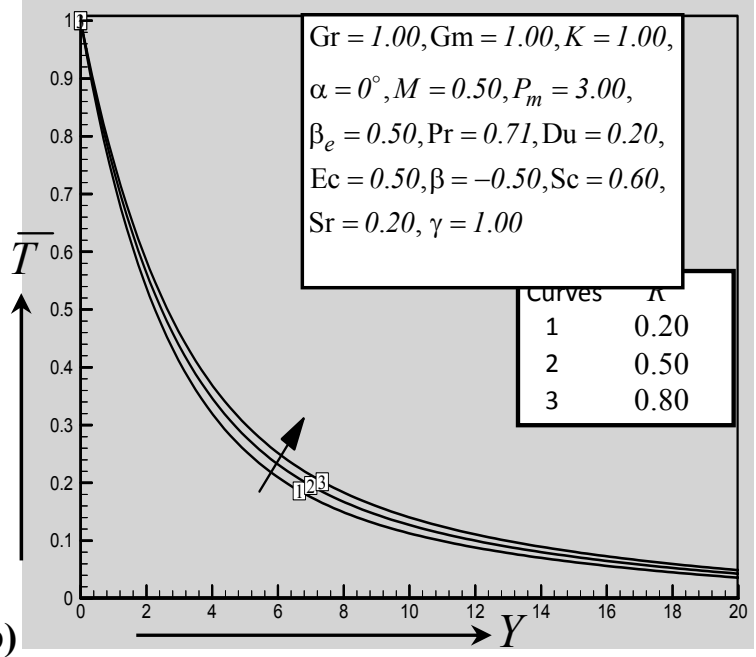

Fig.11. Illustration of temperature profiles for various values of (a) magnetic diffusivity number $\left(P_{m}\right)$ and (b) radiation parameter $(R)$ when $p=2$ and $q=2$.

(a)

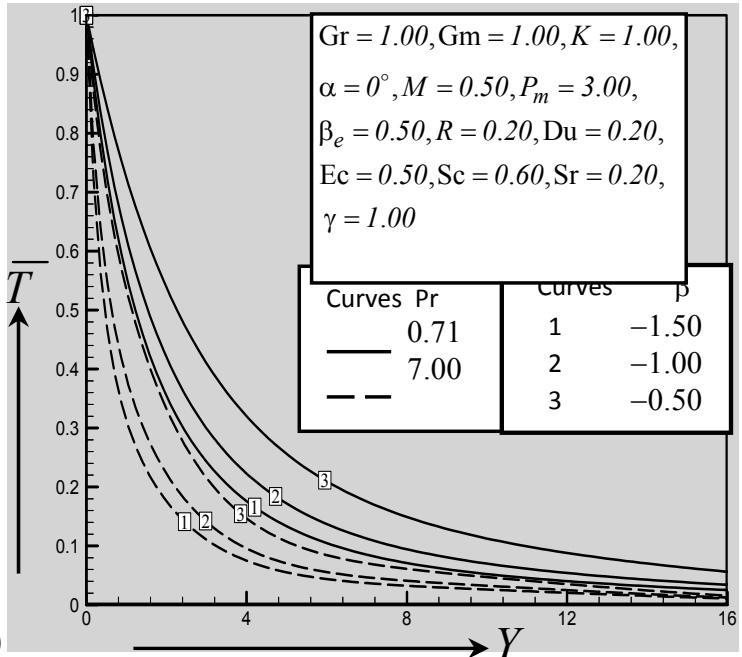

(b)

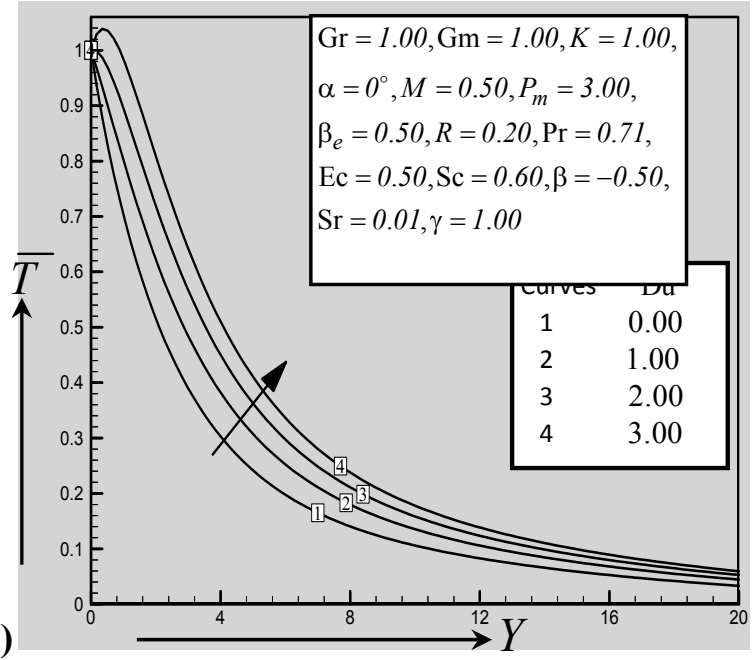

Fig.12. Illustration of temperature profiles for various values of (a) Prandtl number (Pr) and heat genaration or absorption parameter $(\beta)$ and (b) Dufour number $(\mathrm{Du})$ when $p=2$ and $q=2$. 
(a)

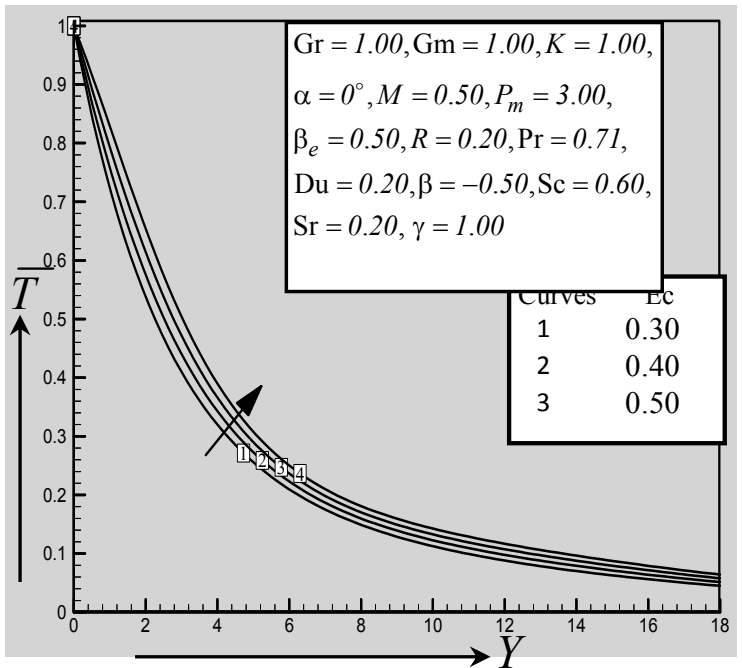

(b)

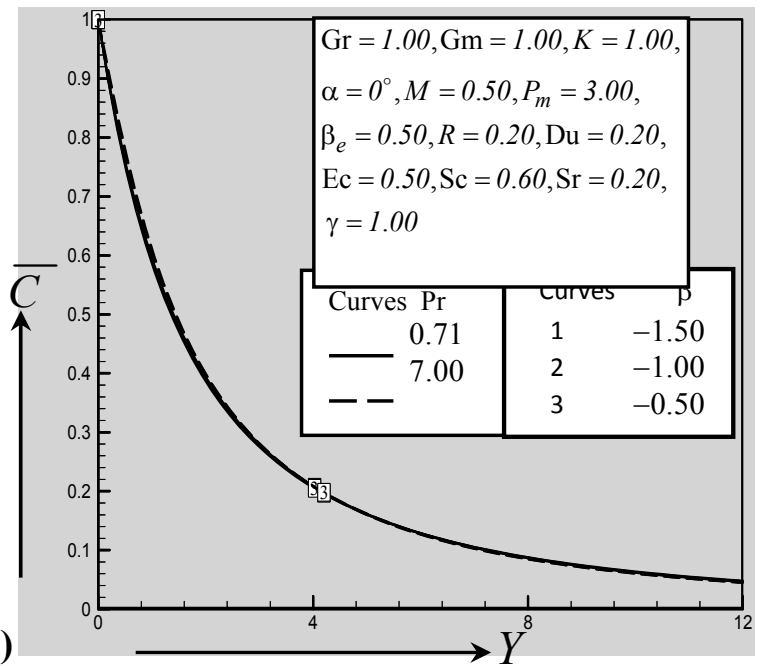

Fig.13. Illustration of (a) temperature profiles for various values of the Eckert number (Ec) and (b) concentration profiles for various values of the Prandtl number (Pr) and heat genaration or absorption parameter $(\beta)$ when $p=2$ and $q=2$.

(a)
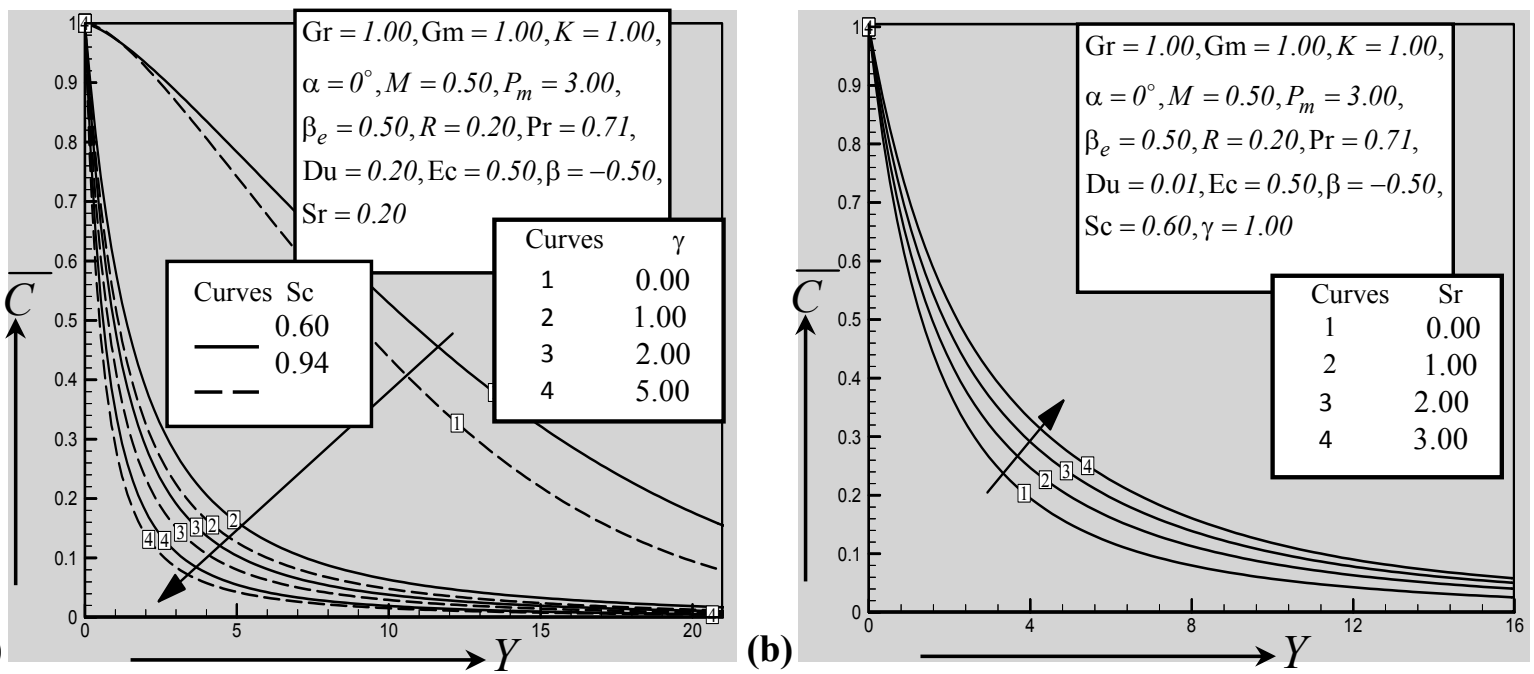

Fig.14. Illustration of concentration profiles for various values of (a) Schmidt number (Sc) and chemical reaction parameter $(\gamma)$ and (b) Soret number (Sr) when $p=2$ and $q=2$.

The average shear stresses in the $x$-direction have been shown for various values of the permeability of the porous medium $(K),(\alpha)$ and magnetic parameter $(M)$, respectively, in Figs 15-16a. These results show that the average shear stress in the $x$-direction decreases with the increase of the permeability of the porous medium and magnetic parameter while opposite effects have been observed with the increase of $(\alpha)$. Figures 16b-17 show the average shear stress in the $z$-direction for various values of the permeability of the porous medium $(K),(\alpha)$ and magnetic parameter $(M)$, respectively. It has been observed that the average shear stress in the $z$-direction decreases with the increase of permeability of the porous medium and $(\alpha)$ while opposite effects have occurred with the increase of the magnetic parameter. The average current densities in the $x$-direction have been displayed for various values of permeability of the porous medium 
$(K),(\alpha)$, magnetic parameter $(M)$, magnetic diffusivity number $\left(P_{m}\right)$ and Hall parameter $\left(\beta_{e}\right)$, respectively in Figs 18-20a. These results show that the average current density in the $x$-direction increases with the increase of the permeability of the porous medium, magnetic parameter and magnetic diffusivity number while opposite effects have occurred with the increase of $(\alpha)$ and the Hall parameter. Figures 20b22 display the average current density in the $z$-direction for various values of the permeability of the porous medium $(K),(\alpha)$, magnetic parameter $(M)$, magnetic diffusivity number $\left(P_{m}\right)$ and Hall parameter $\left(\beta_{e}\right)$ respectively. These results show that the average current density in the $z$-direction decreases with the increase of the permeability of the porous medium, magnetic parameter, magnetic diffusivity number and Hall parameter while increases with the increase of $(\alpha)$. Figures 23-25a display the average Nusselt number for various values of the magnetic diffusivity number $\left(P_{m}\right)$, radiation parameter $(R)$, Prandtl number $(\operatorname{Pr})$ and heat generation or absorption parameter $(\beta)$, Dufour number $(\mathrm{Du})$ and Eckert number $(\mathrm{Ec})$, respectively. These results show that the average Nusselt number decreases with the increase of the radiation parameter, heat generation or absorption parameter, Dufour number and Eckert number, while it increases with the increase of the Prandtl number. It is noted that $\beta<0$ and $\beta>0$ are treated as heat absorption and genaration, respectively. As for the effects of the magnetic diffusivity number, it is observed from Fig. 5.7.21(a) that the average Nusselt number increases within the interval $0<\tau<20$ (approx.), then decreases with an increase of the magnetic diffusivity number. Figures $25 \mathrm{~b}-26$ show the average Sherwood number for several values of the Prandtl number $(\operatorname{Pr})$ and heat generation or absorption parameter $(\beta)$, chemical reaction parameter $(\gamma)$ with two values of the Schmidt number $\mathrm{Sc}=0.60$ (water vapor) and $\mathrm{Sc}=0.94$ (carbon dioxide) and Soret number $(\mathrm{Sr})$, respectively. The average Sherwood number decreases with the increases of the Prandtl number and increases with the increase of the heat generation or absorption parameter. It is noted that the average Sherwood number increases with the increase of the chemical reaction parameter $(\gamma)$, where $\gamma<0$ and $\gamma>0$ are treated as genarative and destructive chemical reaction, respectively. The average Sherwood number also increases with the increase of the Schmidt number. For the effects of the Soret number, it has been observed that the average Sherwood number decreases with the increase of the Soret number.

(a)

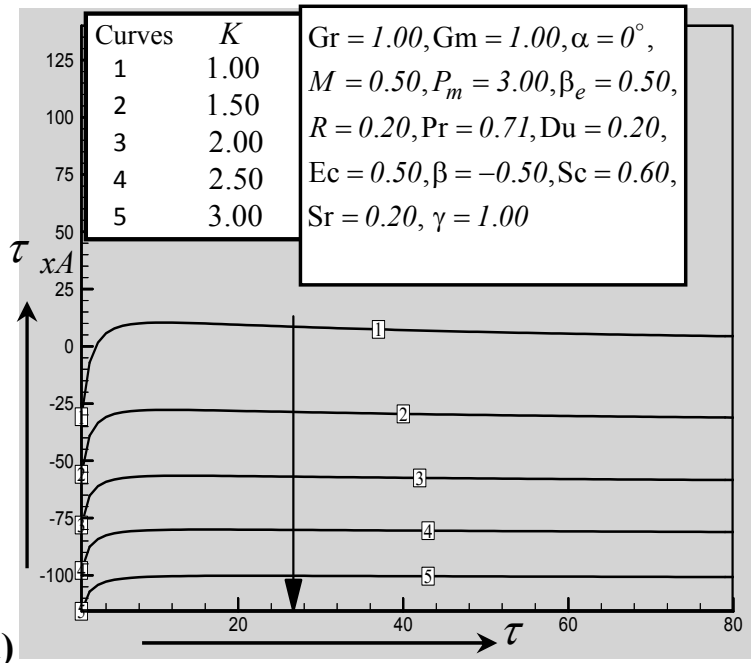

(b)

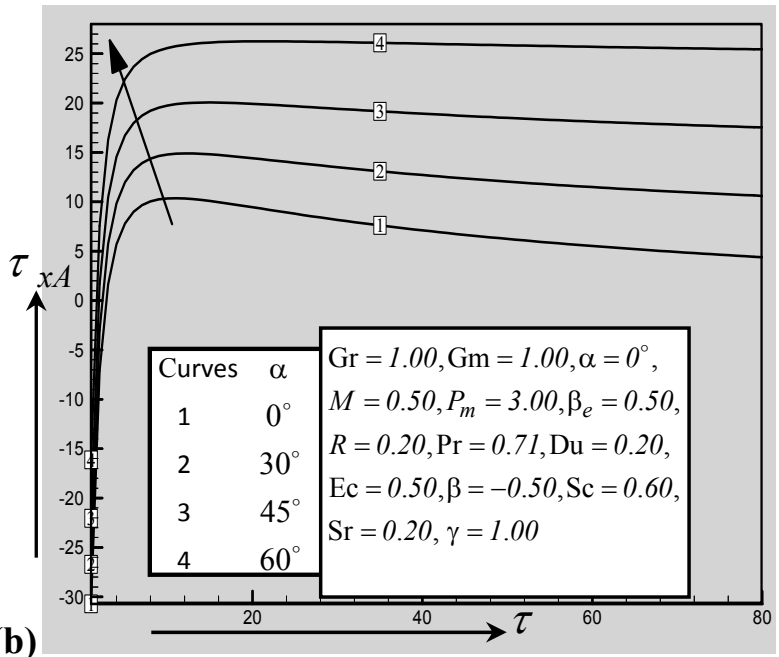

Fig.15. Illustration of average shear stress in the $x$-direction for various values of (a) permeability of the porous medium $(K)$ and (b) $(\alpha)$ when $p=2$ and $q=2$. 
(a)

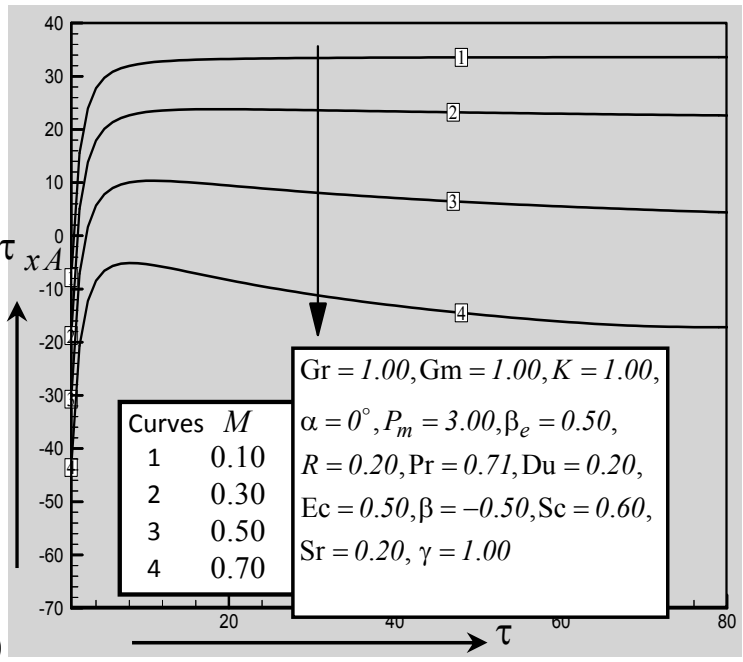

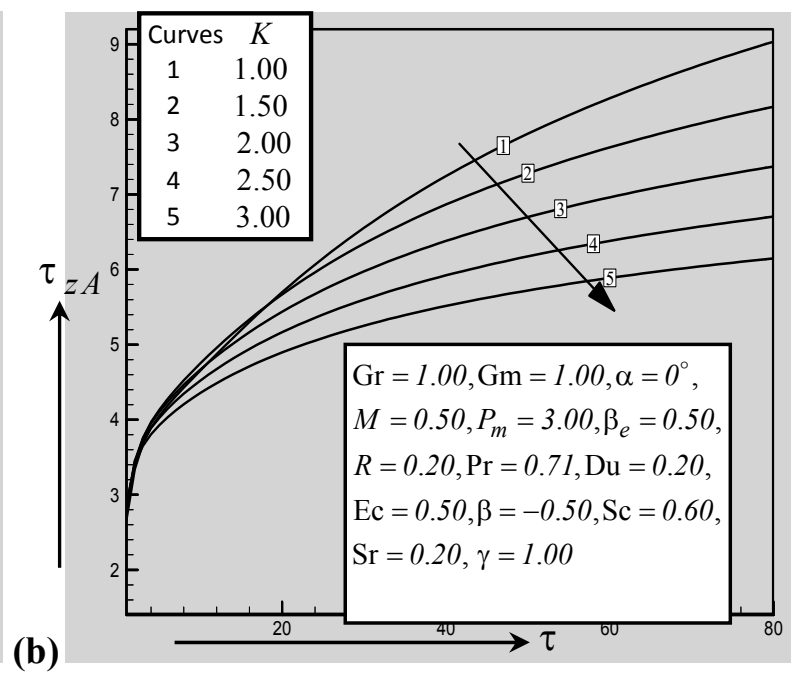

Fig.16. Illustration of (a) average shear stress in the $x$-direction for various values of the magnetic parameter $(M)$ and (b) average shear stress in the $z$-direction for various values of permeability of the porous medium $(K)$ when $p=2$ and $q=2$.
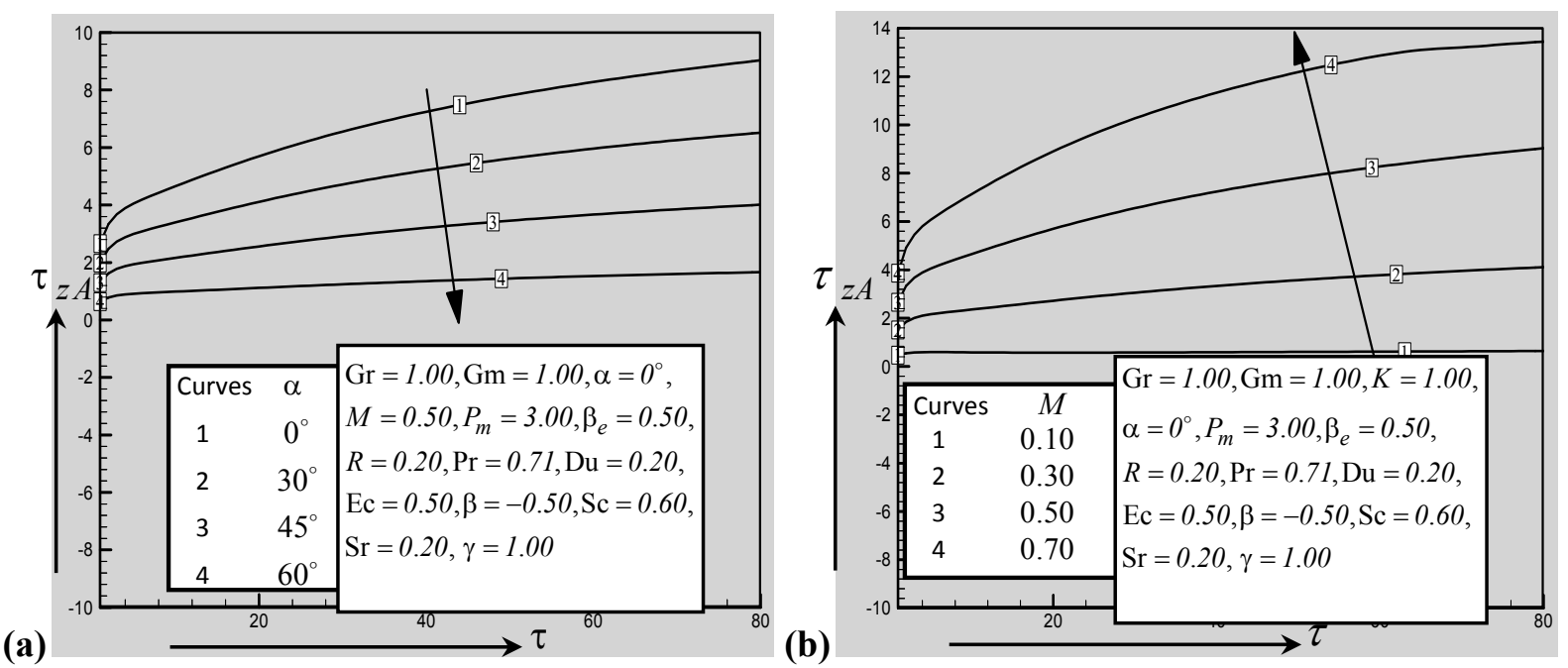

Fig.17. Illustration of average shear stress in the $z$-direction for various values of (a) $(\alpha)$ and (b) magnetic parameter $(M)$ when $p=2$ and $q=2$. 

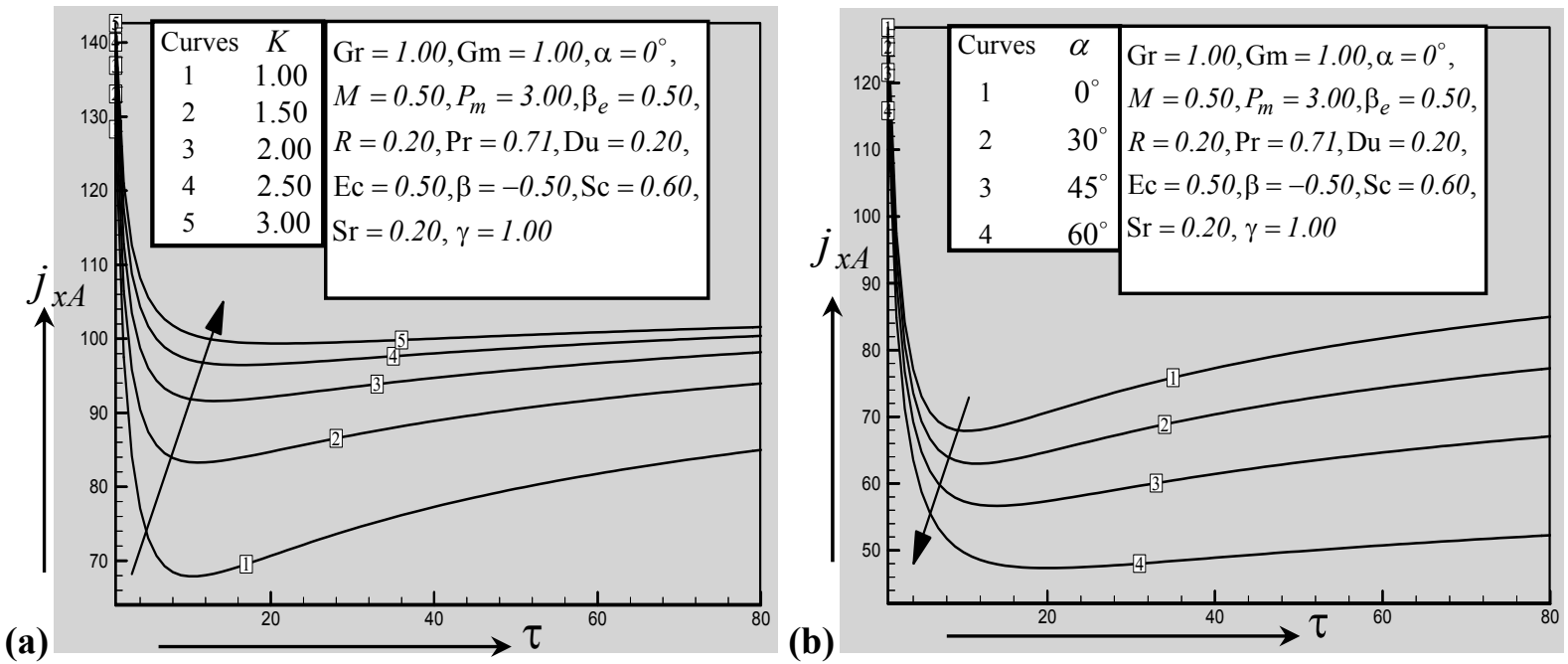

Fig.18. Illustration of average current density in the $x$-direction for various values of (a) permeability of the porous medium $(K)$ and $(b)(\alpha)$ when $p=2$ and $q=2$.
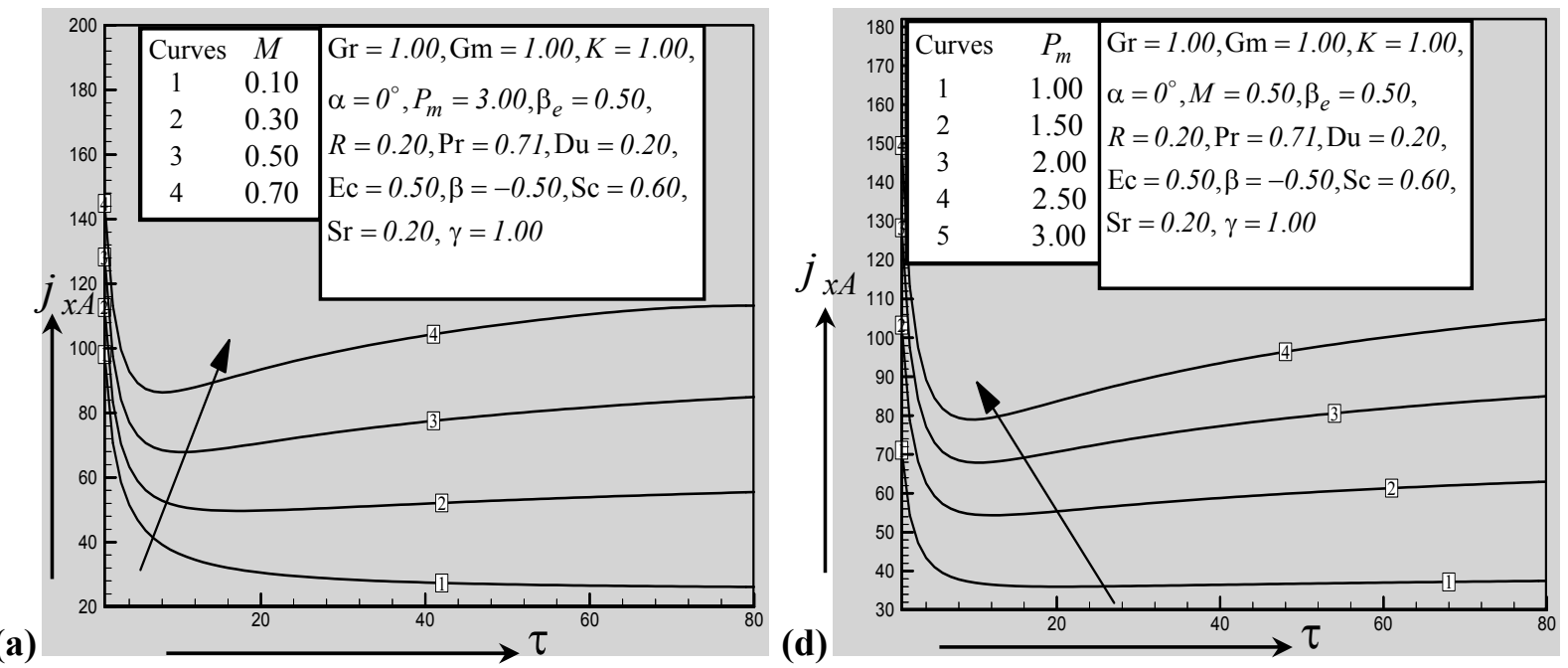

Fig.19. Illustration of average current density in the $x$-direction for various values of (a) magnetic parameter $(M)$ and (b) magnetic diffusivity number $\left(P_{m}\right)$ when $p=2$ and $q=2$. 

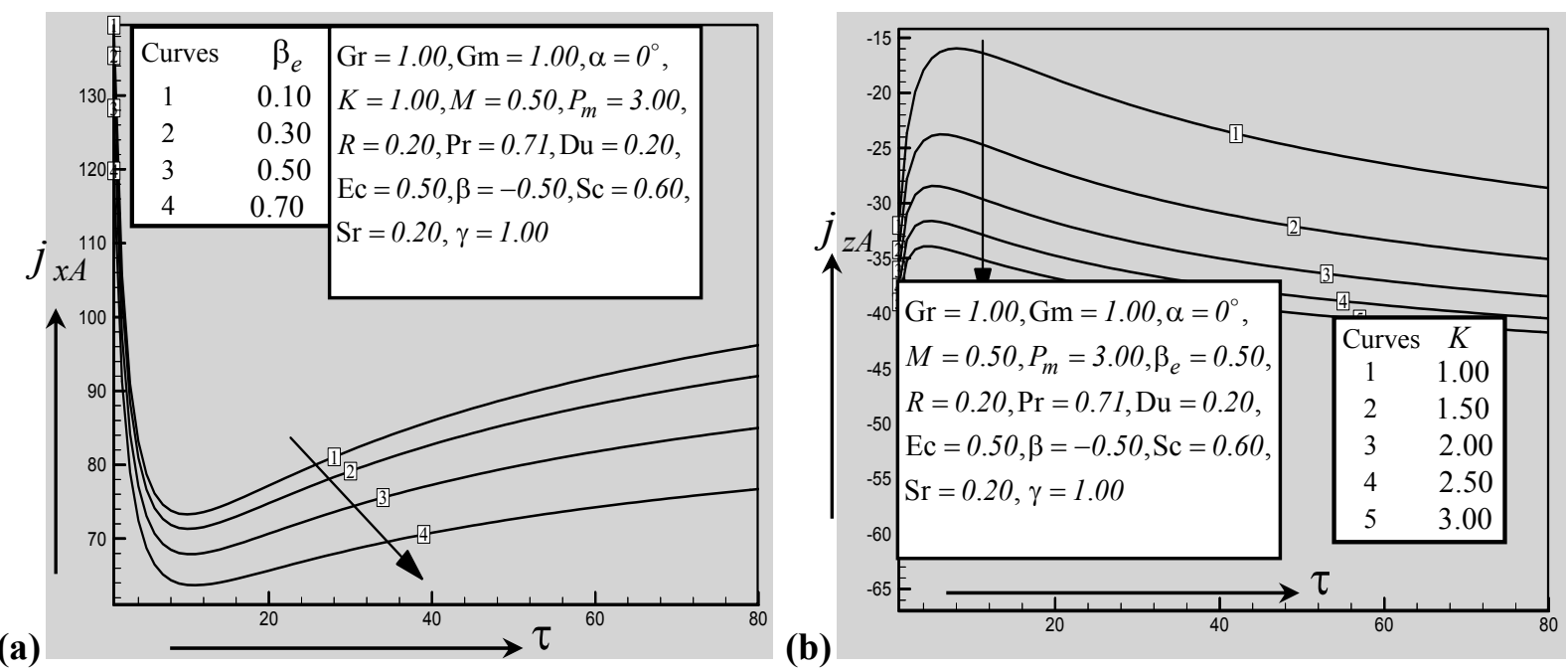

(b)

Fig.20. Illustration of (a) average current density in the $x$-direction for various values of the Hall current parameter $\left(\beta_{e}\right)$ and $(b)$ average current density in the $z$-direction for various values of the permeability of te porous medium $(K)$ when $p=2$ and $q=2$.
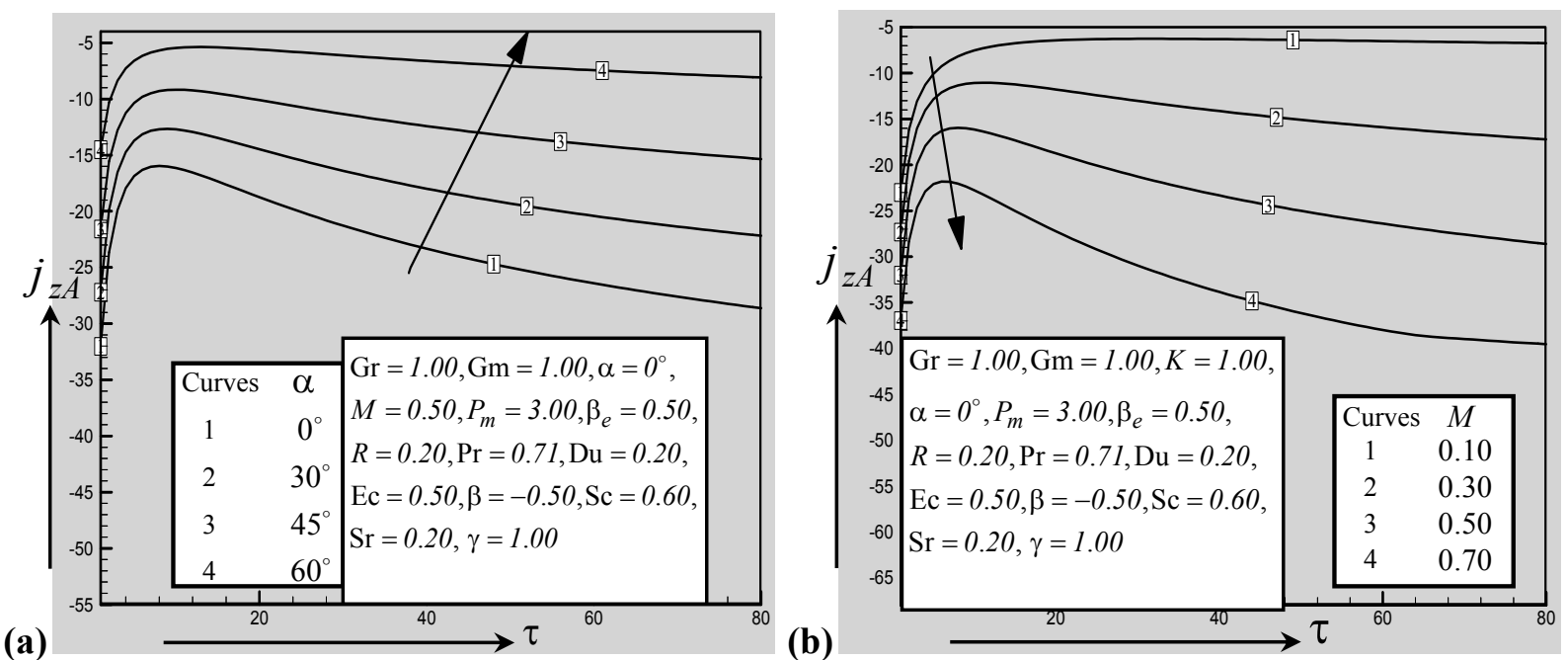

Fig.21. Illustration of average current density in the $z$-direction for various values of (a) ( $\alpha$ ) and (b) magnetic parameter $(M)$ when $p=2$ and $q=2$. 
(a)

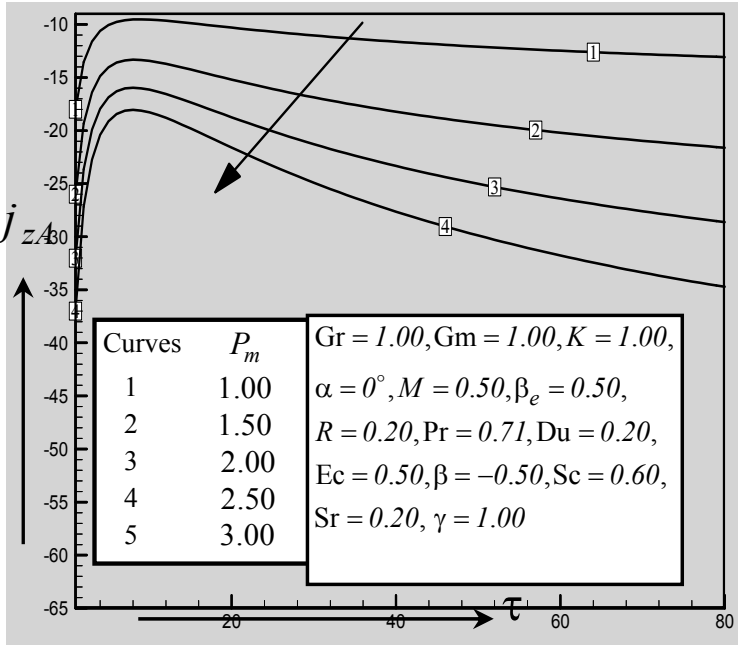

(b)

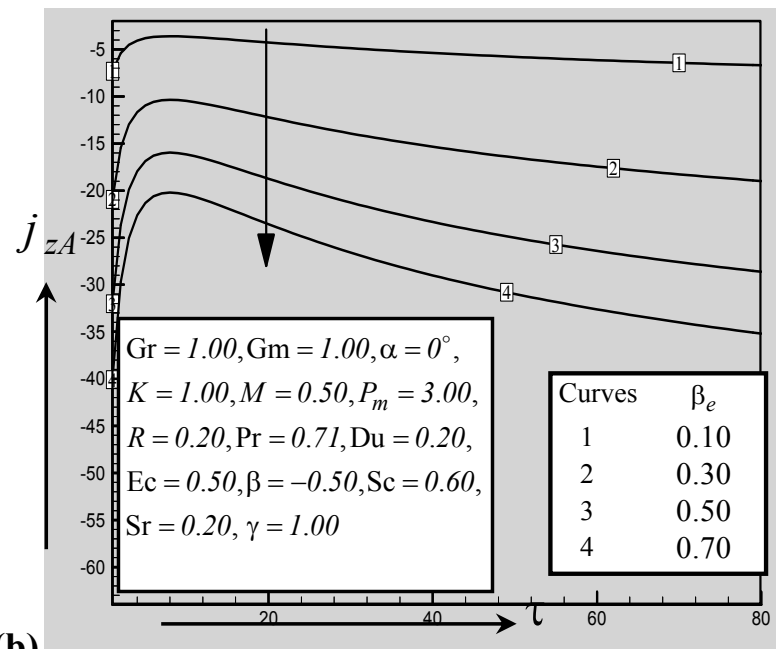

Fig.22. Illustration of average current density in the $z$-direction for various values of (a) magnetic diffusivity number $\left(P_{m}\right)$ and (b) Hall current parameter $\left(\beta_{e}\right)$ when $p=2$ and $q=2$.
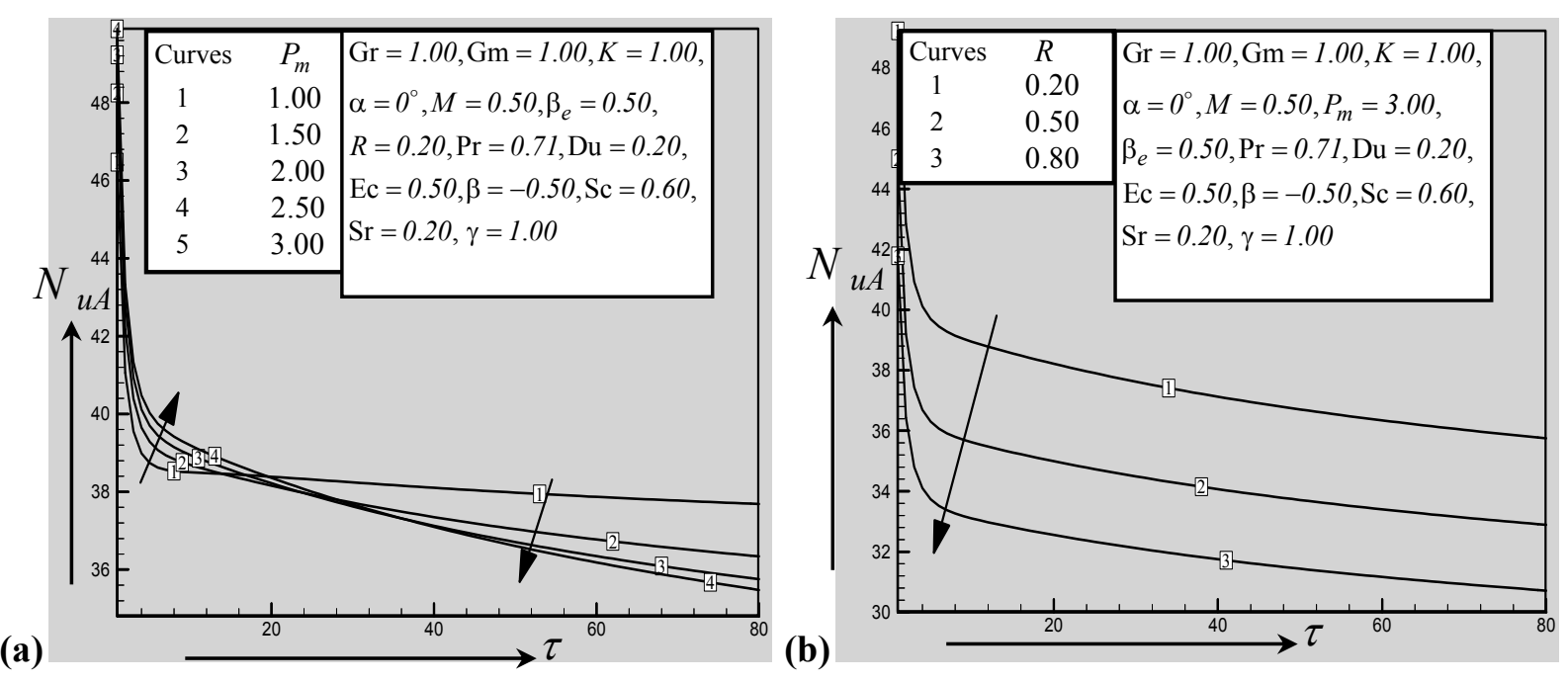

Fig.23. Illustration of average Nusselt number for various values of (a) magnetic diffusivity number $\left(P_{m}\right)$ and (b) radiation parameter $(R)$ when $p=2$ and $q=2$. 

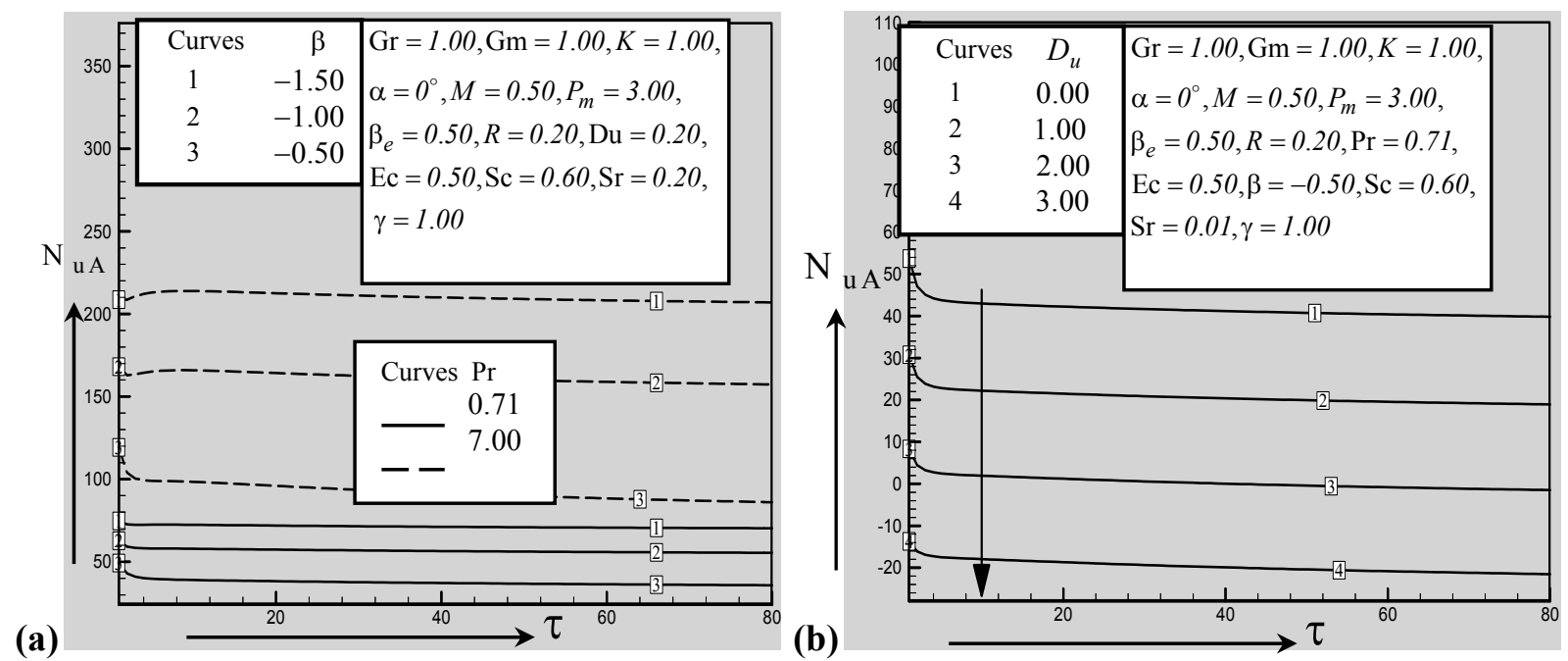

Fig.24. Illustration of average Nusselt number for various values of (a) Prandtl number (Pr) and heat genaration or absorption parameter $(\beta)$ and (b) Dufour number (Du) when $p=2$ and $q=2$.
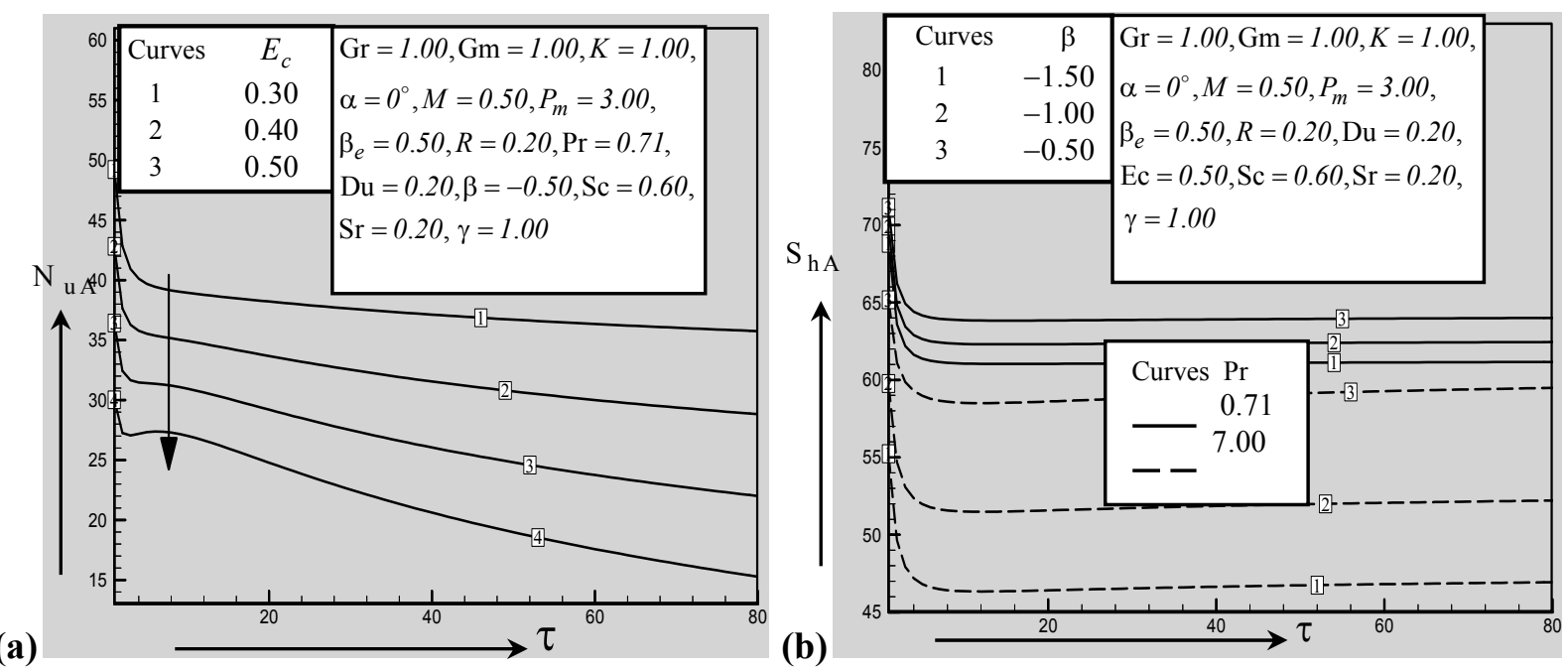

Fig.25. Illustration of (a) average Nusselt number for various values of the Eckert number (Ec) and (b) average Sherwood number for various values of the Prandtl number $(\operatorname{Pr})$ and heat genaration or absorption parameter $(\beta)$ when $p=2$ and $q=2$. 
(a)

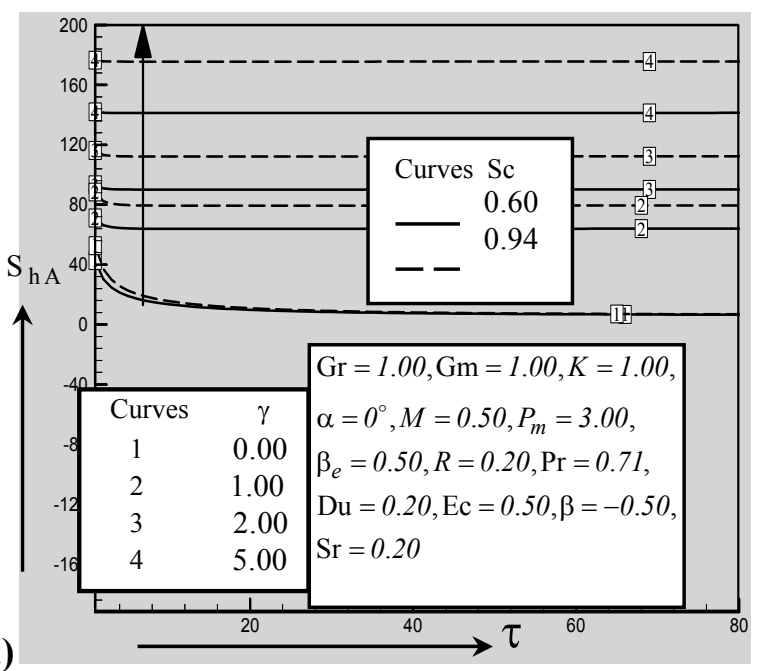

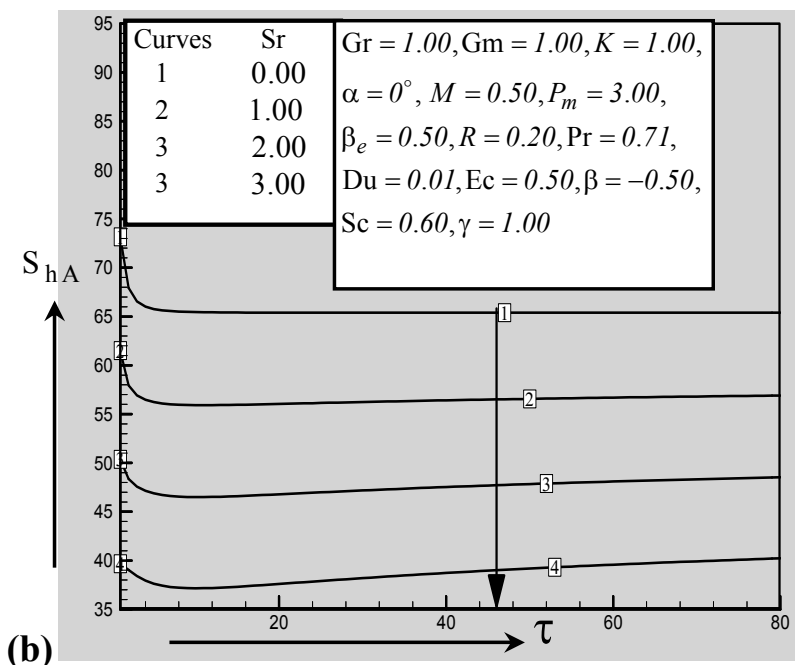

Fig.26. Illustration of average Sherwood number for various values of (a) Schmidt number (Sc) and chemical reaction parameter $(\gamma)$ (b) Soret number $(\mathrm{Sr})$ when $p=2$ and $q=2$.

The local shear stresses in the $x$-direction have been shown for various values of the permeability of the porous medium $(K),(\alpha)$ and magnetic parameter $(M)$, respectively, in Figs 27-28a. These results show that the local shear stress in the $x$-direction decreases with the increase of the permeability of the porous medium and magnetic parameter while opposite effects have occurred with the increase of $(\alpha)$. Figures 28b-29 show the local shear stress in the $z$-direction for various values of the permeability of the porous medium $(K),(\alpha)$ and magnetic parameter $(M)$, respectively. It has been observed that the local shear stress in the $z$-direction decreases with the increase of permeability of the porous medium and $(\alpha)$ while opposite effects have been observed with the increase of the magnetic parameter. The local current densities in the $x$-direction have been illustrated for various values of the permeability of the porous medium $(K),(\alpha)$, magnetic parameter $(M)$, magnetic diffusivity number $\left(P_{m}\right)$ and Hall parameter $\left(\beta_{e}\right)$ respectively, in Figs 30-32a. These results show that the local current density in the $x$-direction increases with the increase of the permeability of the porous medium, magnetic parameter and magnetic diffusivity number while opposite effects have been observed with the increase of $(\alpha)$ and Hall parameter. Figures 32b34 display the local current density in the $z$-direction for various values of the permeability of the porous medium $(K),(\alpha)$, magnetic parameter $(M)$, magnetic diffusivity number $\left(P_{m}\right)$ and Hall parameter $\left(\beta_{e}\right)$ respectively. These results show that the local current density in the $z$-direction decreases with the increase of the permeability of the porous medium, magnetic parameter, magnetic diffusivity number and Hall parameter while opposite effects have been observed with the increase of $(\alpha)$.

Figures 35-37a display the local Nusselt number for various values of the magnetic diffusivity number $\left(P_{m}\right)$, radiation parameter $(R)$, Prandtl number $(\operatorname{Pr})$ and heat generation or absorption parameter $(\beta)$, Dufour number $(\mathrm{Du})$ and Eckert number $(\mathrm{Ec})$, respectively. These results show that the local Nusselt number decreases with the increase of the heat generation or absorption parameter, Dufour number and Eckert number while increases with the increase of the Prandtl number, where $\beta<0$ and $\beta>0$ are treated as heat absorption and generation, respectively. As for the effects of the magnetic diffusivity number and radiation parameter, it is observed from Fig. 35 that there is no change of the local Nusselt number. 
Figures 37b-39 display the local Sherwood number for several values of the Prandtl number (Pr) and heat generation or absorption parameter $(\beta)$, chemical reaction parameter $(\gamma)$ with two values of Schimdt number $\mathrm{Sc}=0.60$ (water vapor) and $\mathrm{Sc}=0.94$ (carbon dioxide) and Soret number ( $\mathrm{Sr}$ ), respectively. The local Sherwood number decreases with the increases of the Prandtl number while there iss no change with the increase of the heat generation or absorption parameter. It is noted that the local Sherwood number increases with the increase of the chemical reaction parameter $(\gamma)$, where $\gamma<0$ and $\gamma>0$ are treated as generative and destructive chemical reaction, respectively. The local Sherwood number also increases with the increase of the Schimdt number. For the effects of the Soret number, it has been observed that the local Sherwood number decreases with the increase of Soret number.

(a)

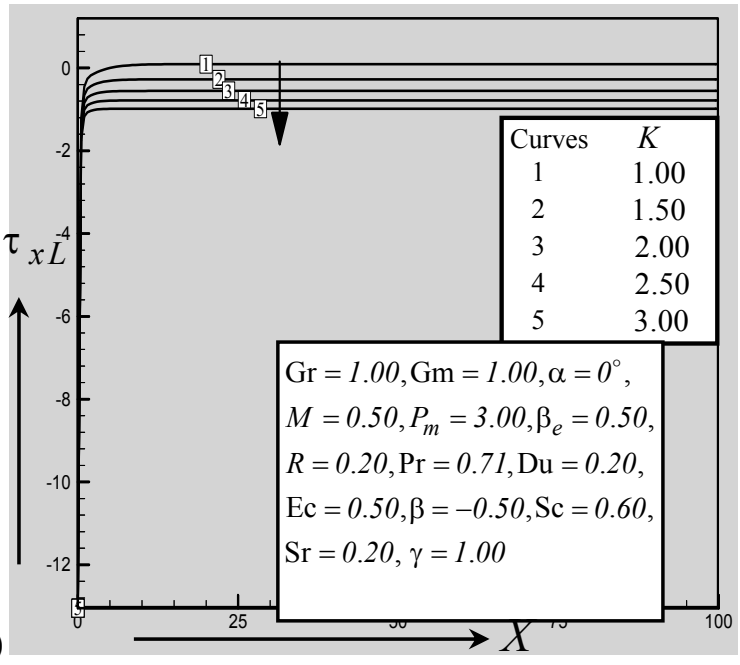

(b)

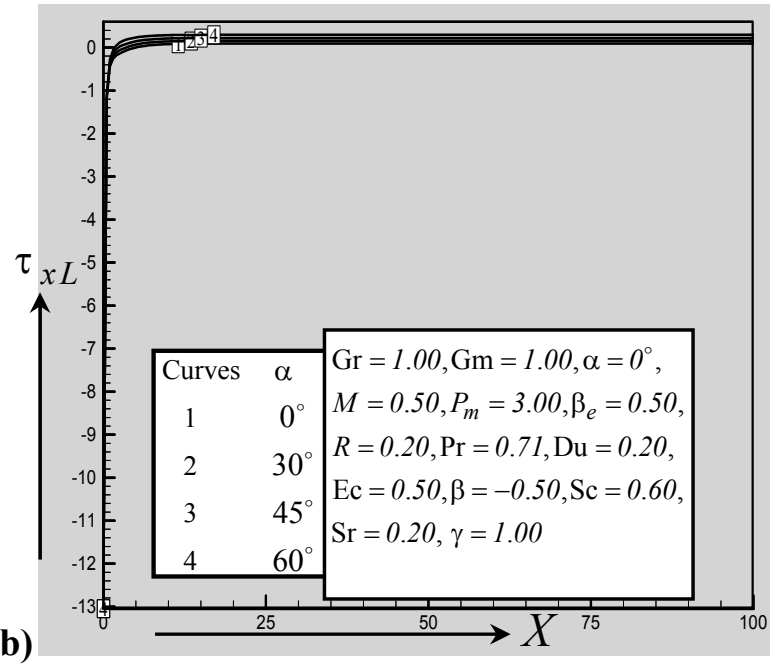

Fig.27. Illustration of local shear stress in the $x$-direction for various values of (a) permeability of the porous medium $(K)$ and (b) $(\alpha)$ when $p=2$ and $q=2$.

(a)

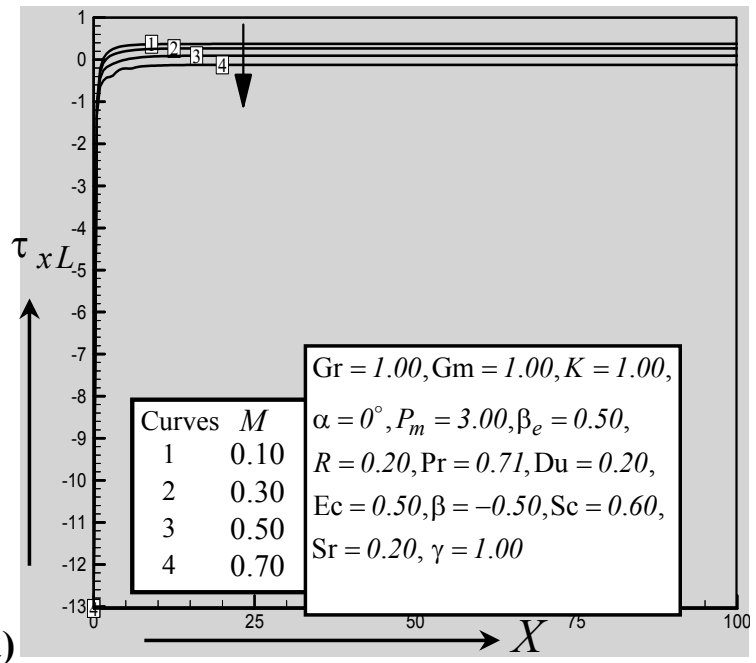

(b)

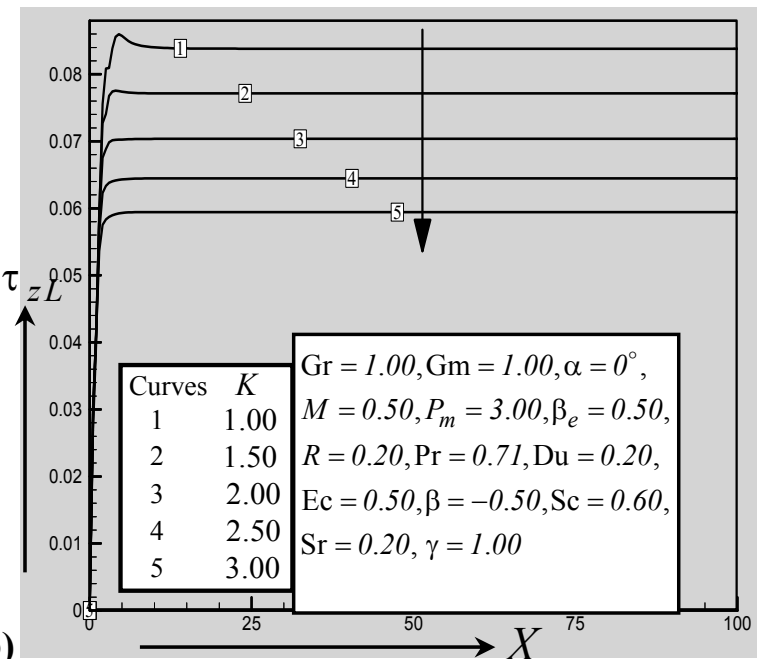

Fig.28. Illustration of (a) local shear stress in the $x$-direction for various values of the magnetic parameter $(M)$ and (b) local shear stress in the $z$-direction for various values of the permeability of the porous medium $(K)$ when $p=2$ and $q=2$. 

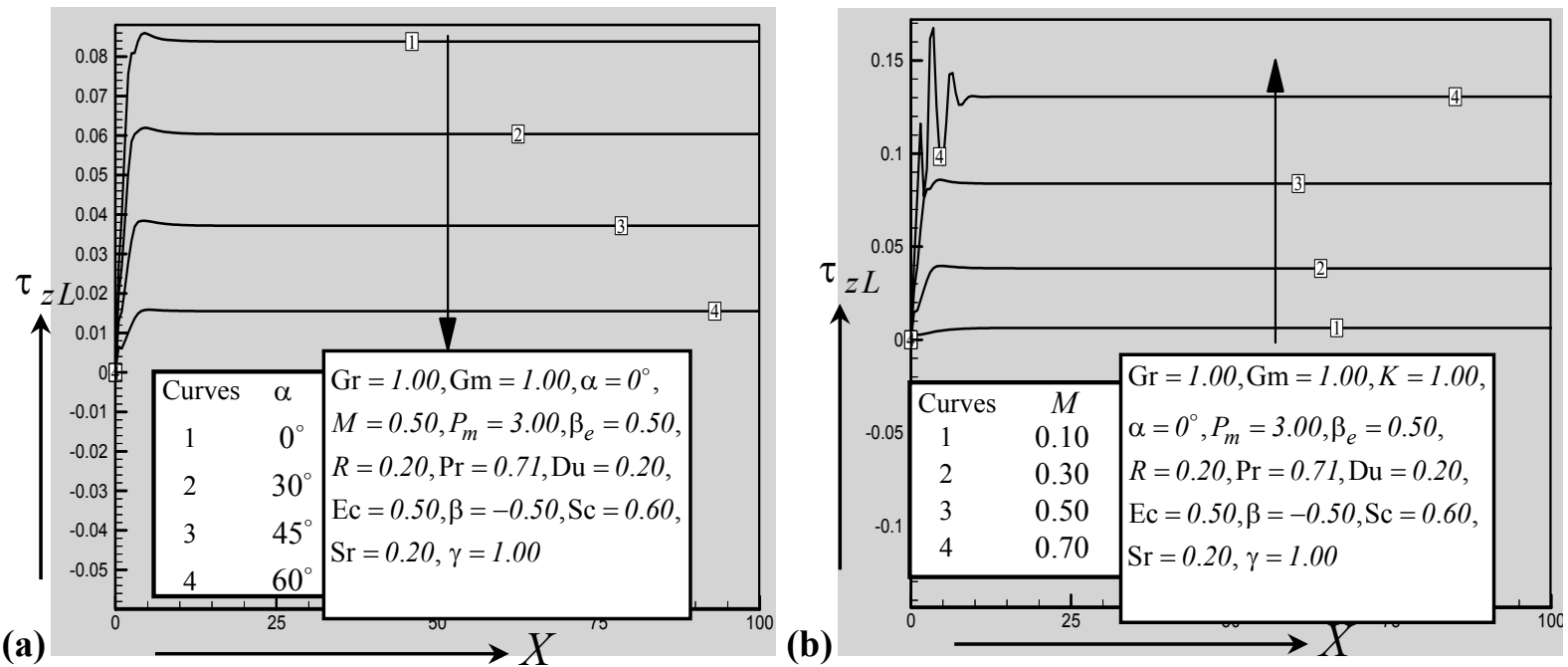

Fig.29. Illustration of local shear stress in the $z$-direction for various values of (a) $(\alpha)$ and (b) magnetic parameter $(M)$ when $p=2$ and $q=2$.
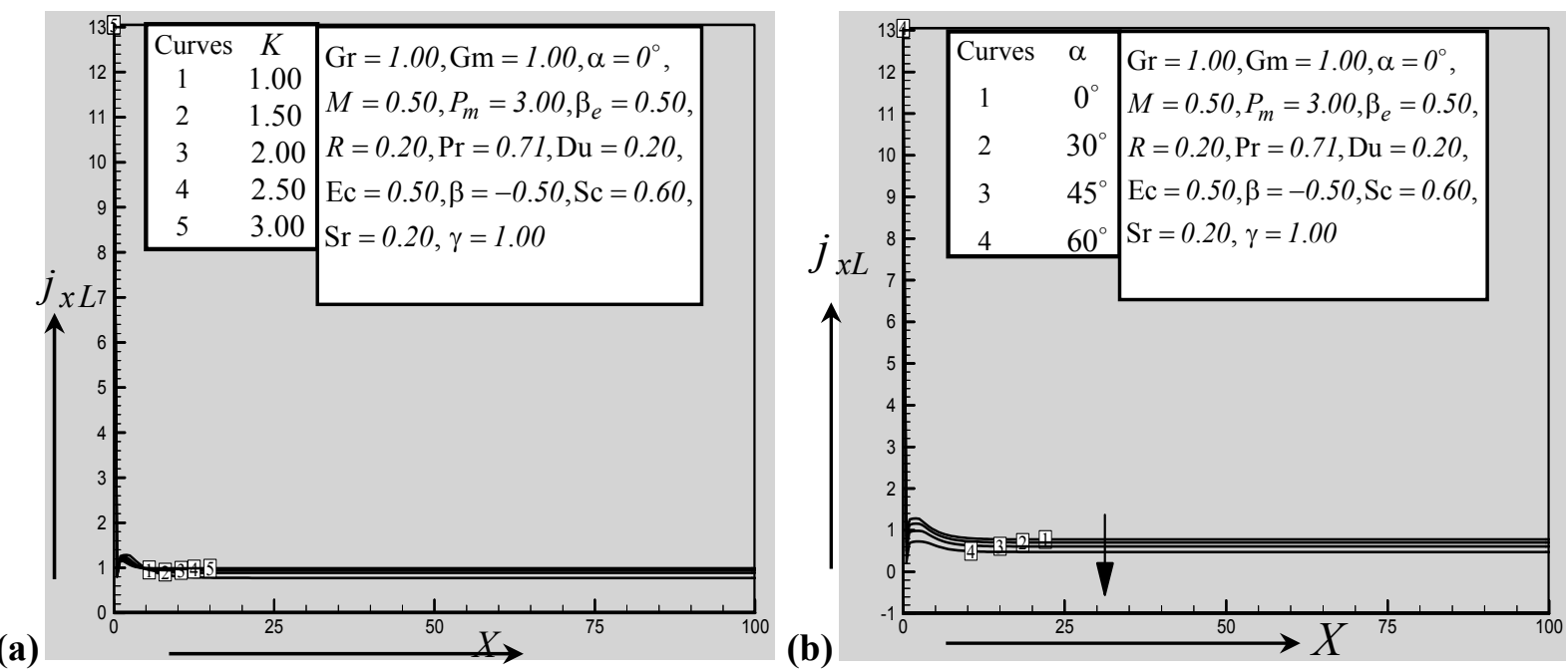

Fig.30. Illustration of local current density in the $x$-direction for various values of (a) permeability of the porous medium $(K)$ and $(\mathrm{b})(\alpha)$ when $p=2$ and $q=2$. 
(a)
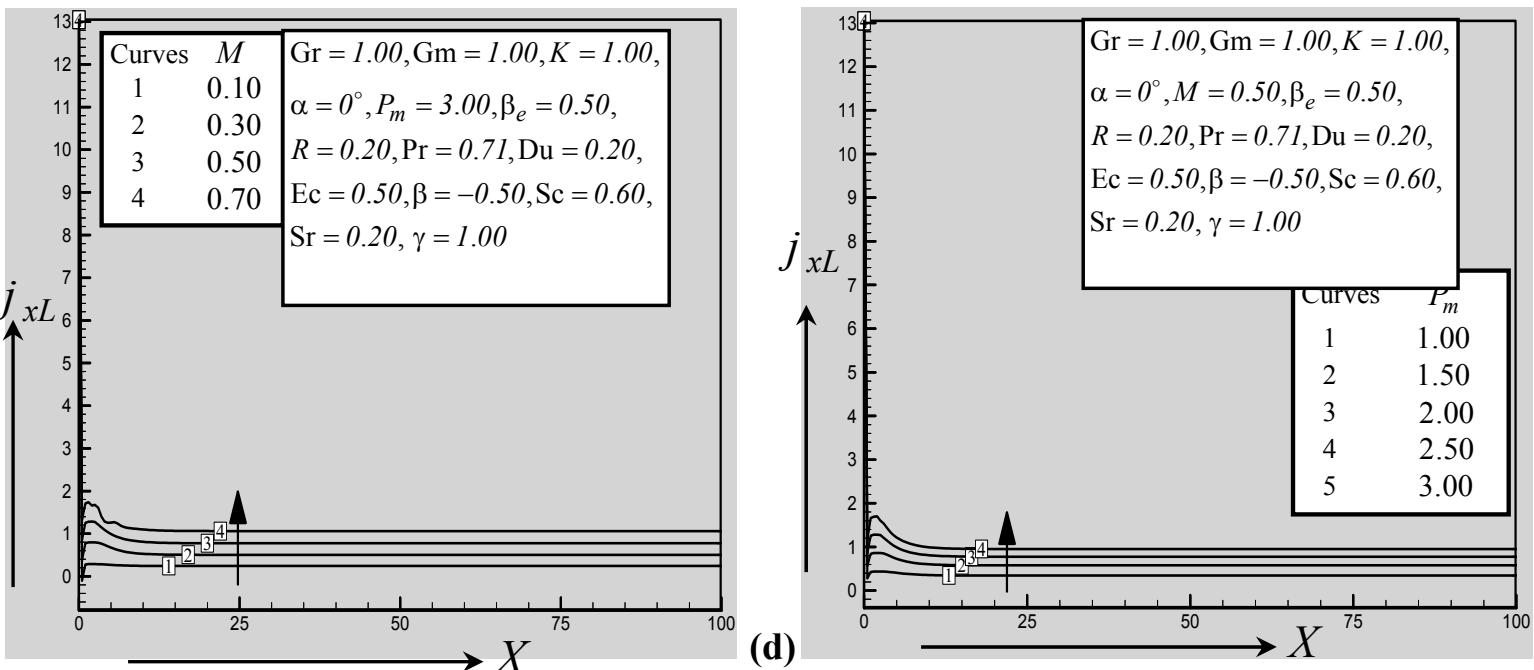

Fig.31. Illustration of local current density in the $x$-direction for various values of (a) magnetic parameter $(M)$ and (b) magnetic diffusivity number $\left(P_{m}\right)$ when $p=2$ and $q=2$.
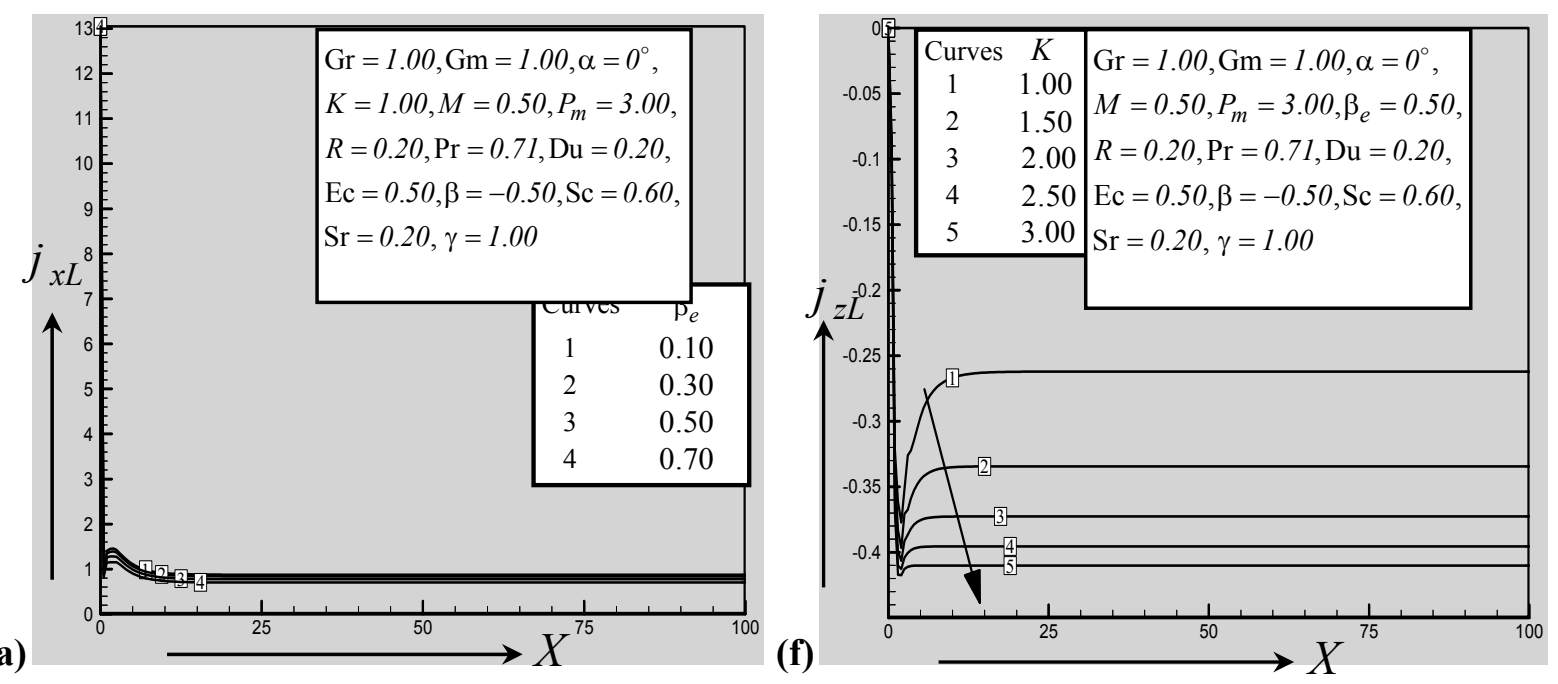

Fig.32. Illustration of (a) local current density in the $x$-direction for various values of the Hall current parameter $\left(\beta_{e}\right)$ and (b) local current density in the $x$-direction for various values values of the permeability of the porous medium $(K)$ when $p=2$ and $q=2$. 

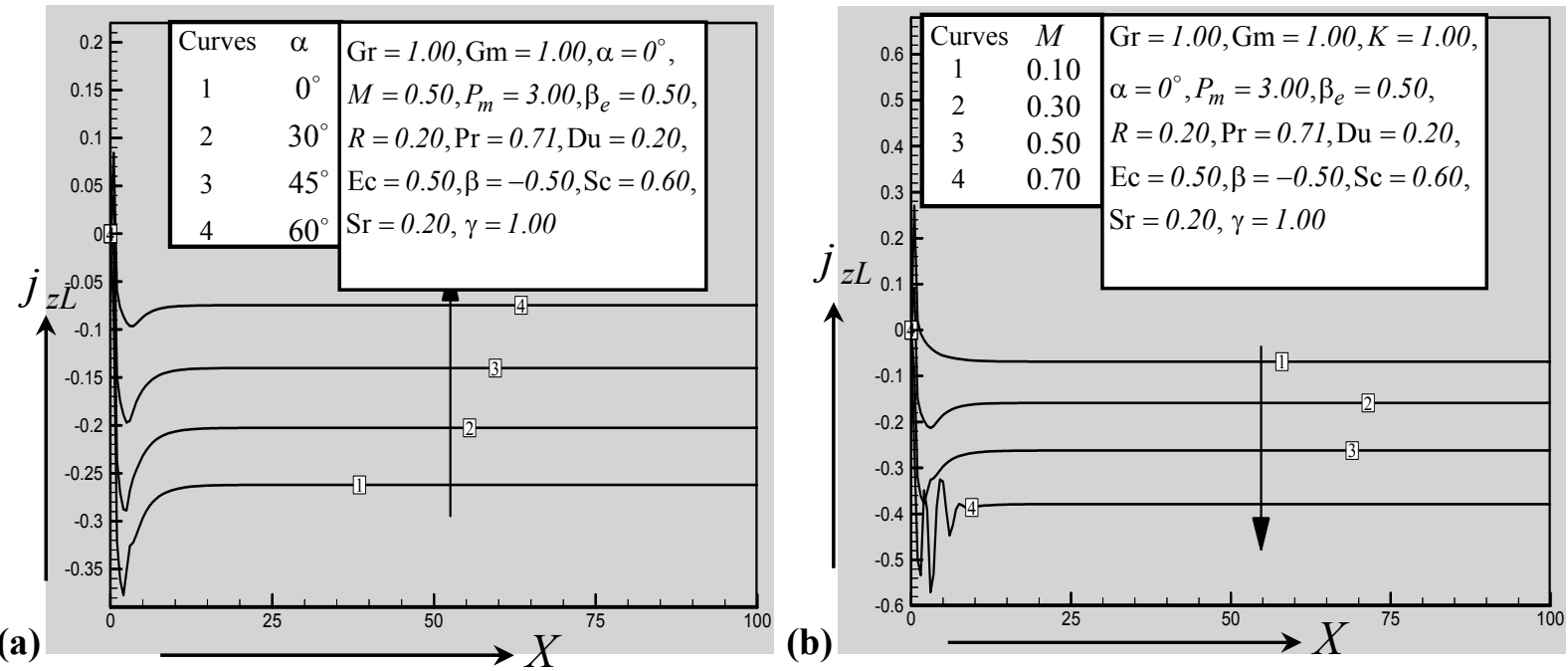

Fig.33. Illustration of average current density in the $z$-direction for various values of (a) ( $\alpha$ ) and (b) magnetic parameter $(M)$ when $p=2$ and $q=2$.

(a)

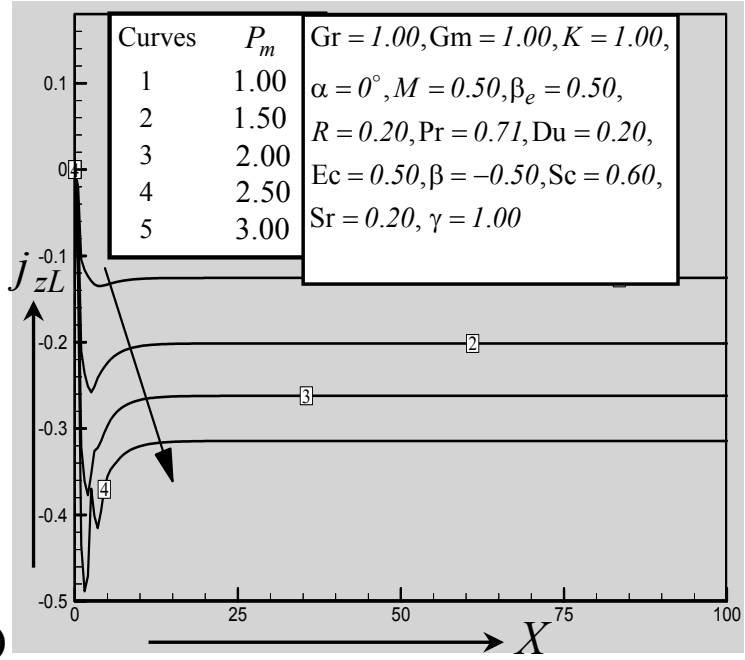

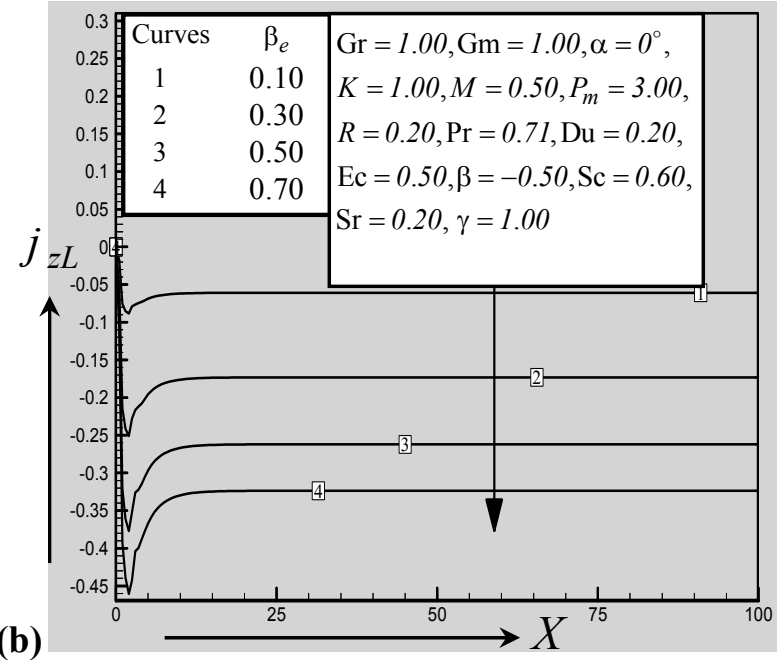

(b)

Fig.34. Illustration of local current density in the $z$-direction for various values of (a) magnetic diffusivity number $\left(P_{m}\right)$ and (b) Hall current parameter $\left(\beta_{e}\right)$ when $p=2$ and $q=2$. 

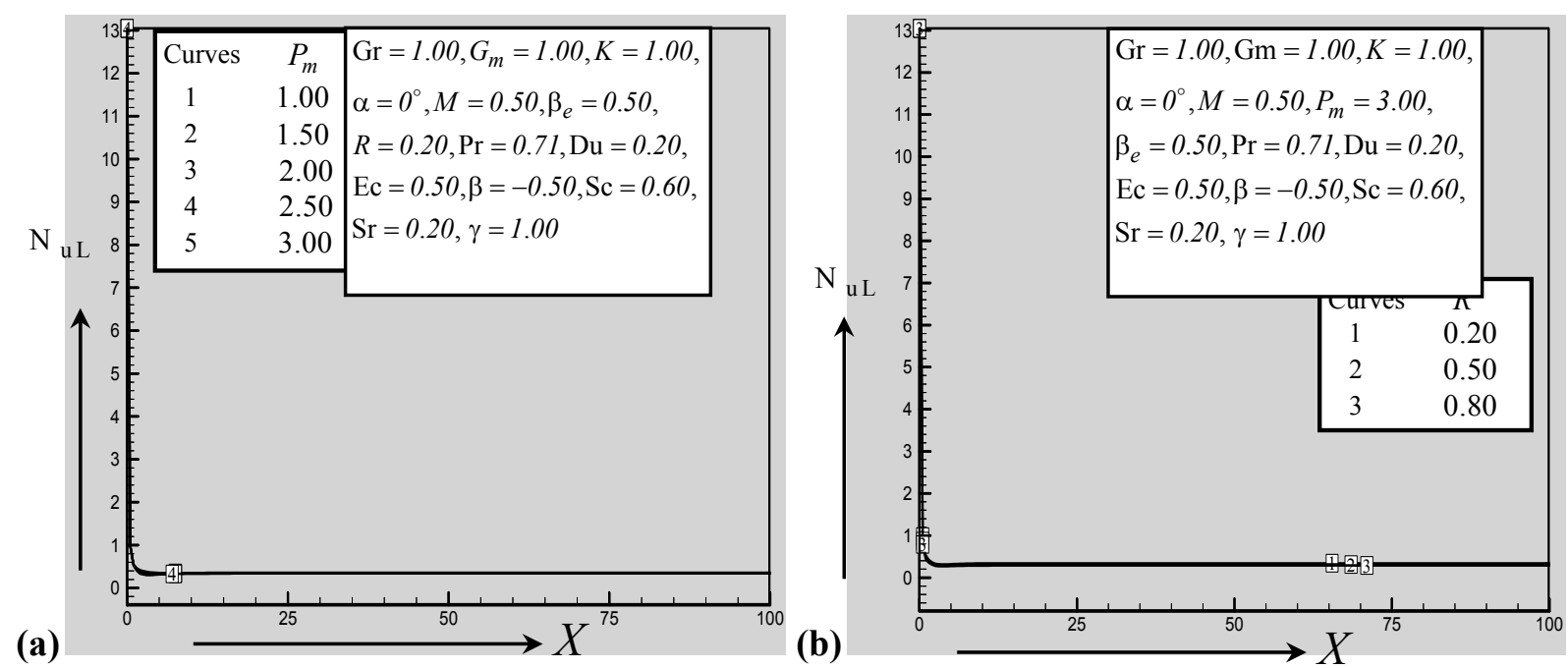

Fig.35. Illustration of the local Nusselt number for various values of (a) magnetic diffusivity number $\left(P_{m}\right)$ and (b) radiation parameter $(R)$ when $p=2$ and $q=2$.
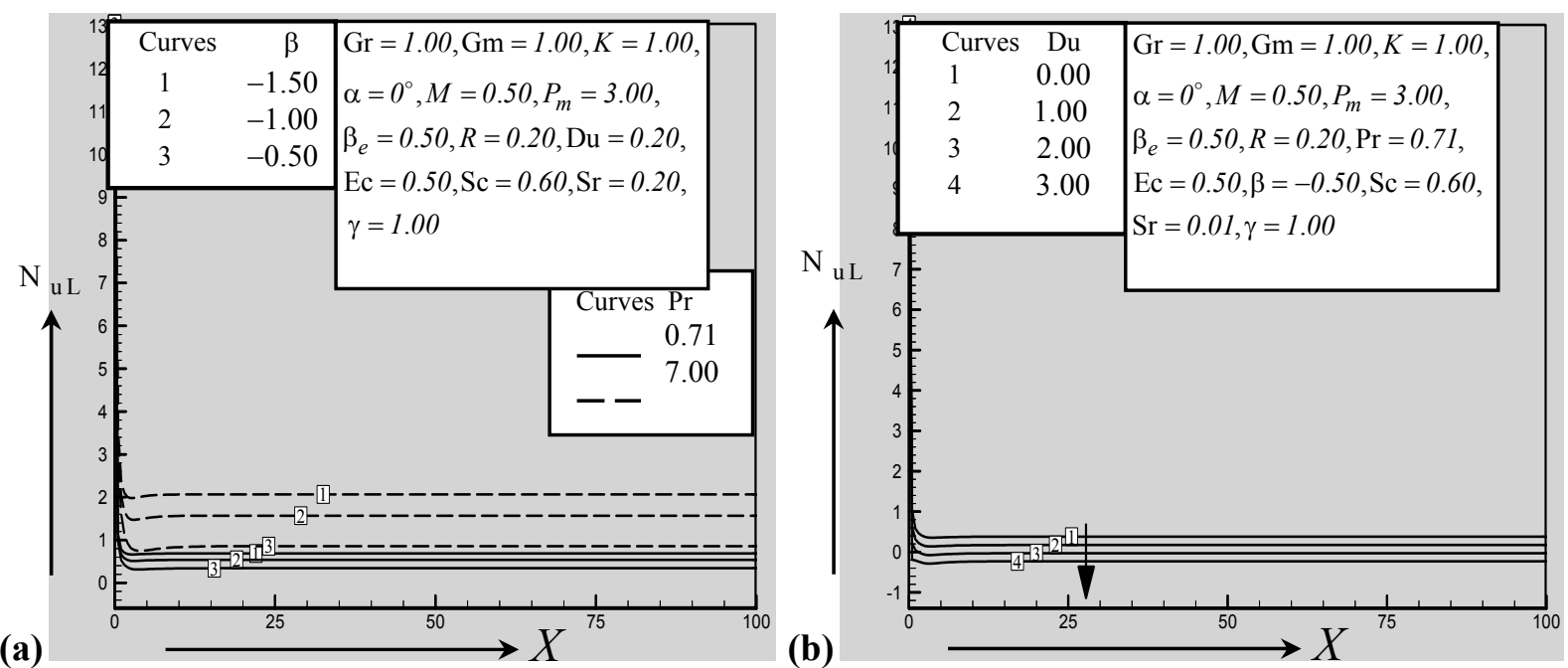

Fig.36. Illustration of local Nusselt number for various values of (a) Prandtl number (Pr) and heat genaration or absrption parameter $(\beta)$ and (b) Dufour number $(\mathrm{Du})$ when $p=2$ and $q=2$. 
(a)
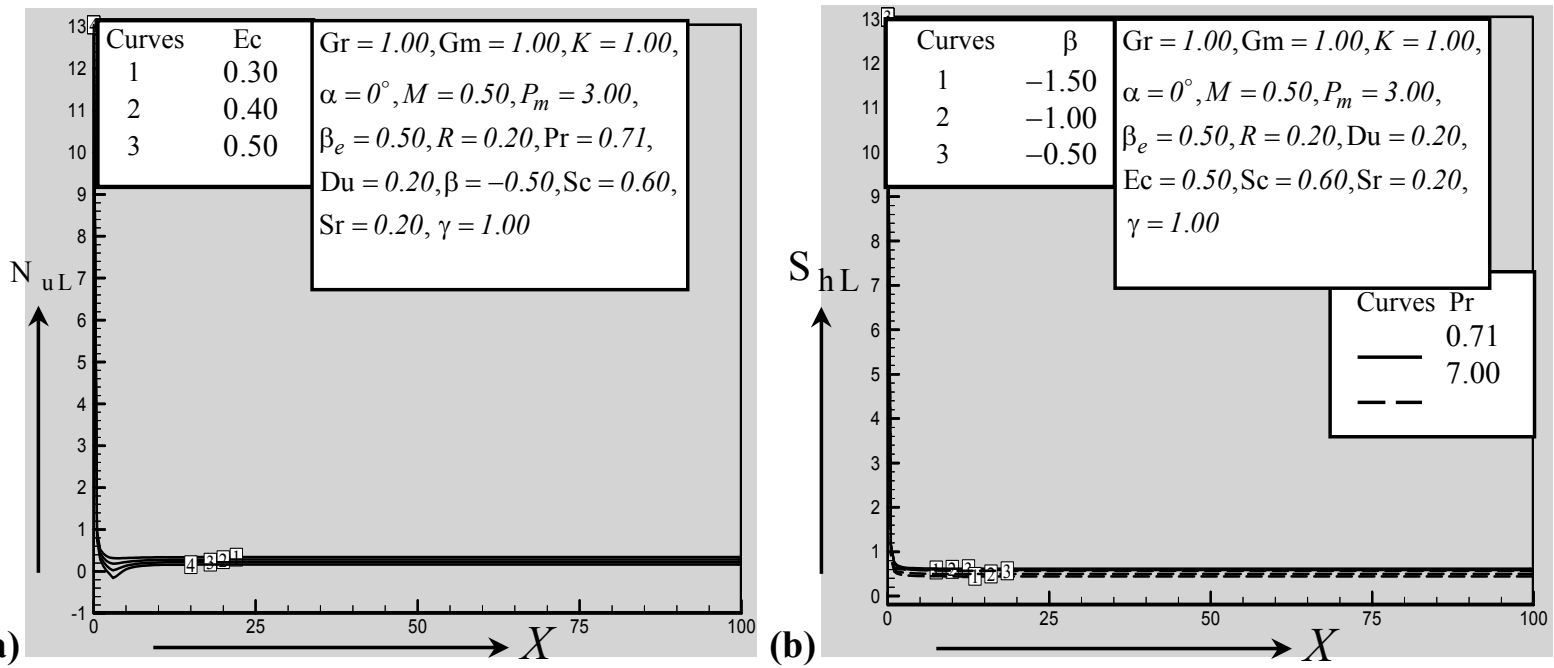

Fig.37. Illustration of (a) local Nusselt number for various values of the Eckert number (Ec) and (b) local Sherwood number for various values of the Prandtl number (Pr) and heat genaration or absrption parameter $(\beta)$ when $p=2$ and $q=2$.
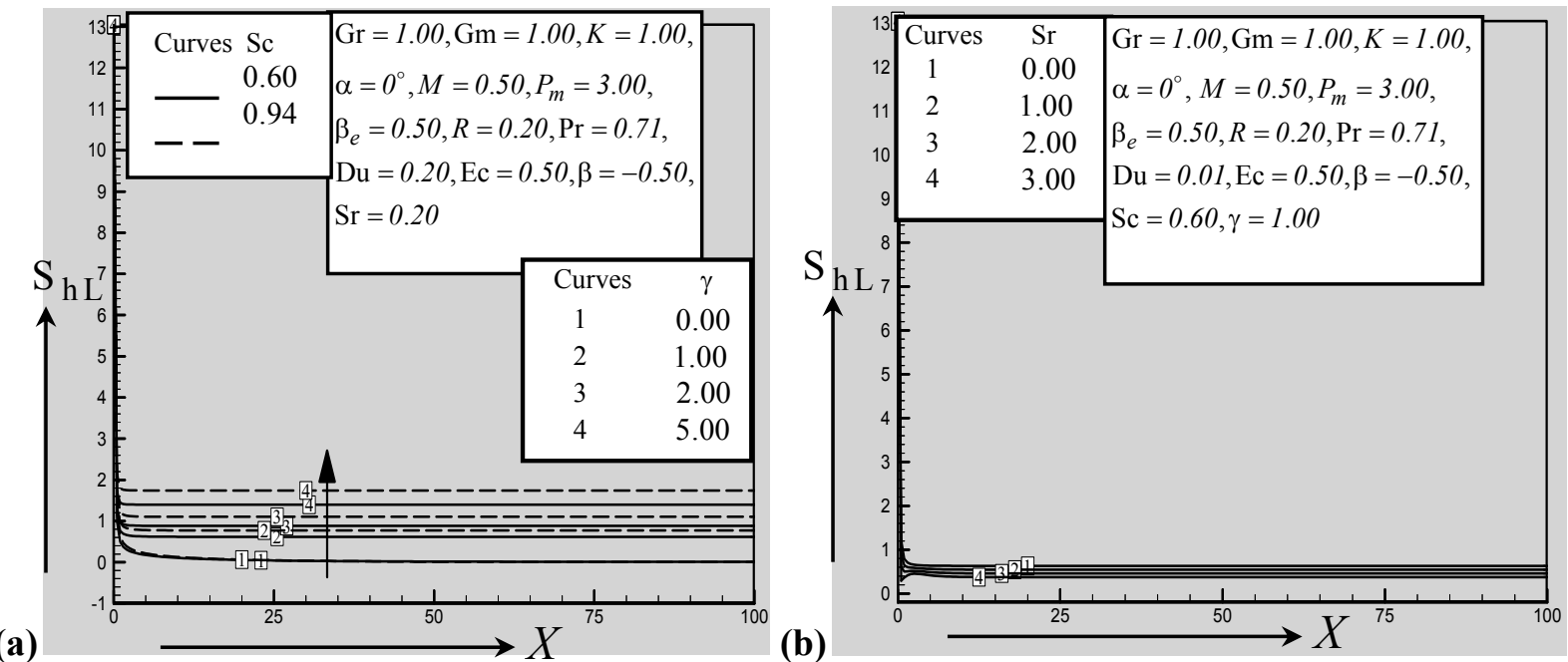

Fig.38. Illustration of the local Sherwood number for various values of (a) Schmidt number (Sc) and chemical reaction parameter $(\gamma)$ and (b) Soret number $(\mathrm{Sr})$ when $p=2$ and $q=2$.

\section{Conclusions}

The explicit finite difference solution for considerable magnetic Reynolds number of unsteady chemically reacting radiative ionized fluid flow over an impulsive vertical porous plate in the presence of heat generation, and Joule heating for $p \leq 2$ and $q \leq 2$ has been investigated. The physical properties are graphically discussed for different values of corresponding parameters and our steady-state results are compared with Haque et al. [12]. The accuracy of our results is qualitatively good in for all the flow parameters.

Some important findings of this investigation are listed below;

1. The average and local shear stress in the $x$-direction decreases with the increase of the permeability of 
the porous medium and magnetic parameter, while it increases with the increase of $(\alpha)$.

2. The average and local shear stress in the $z$-direction decreases with the increase of the permeability of the porous medium and $(\alpha)$ whereas it increases with the increase of the magnetic parameter.

3. The average and local current density in the $x$-direction increases with the increase of the permeability of the porous medium, magnetic parameter and magnetic diffusivity number, while it decreases with the increase of $(\alpha)$ and Hall parameter.

4. The average and local current density in the $z$-direction decreases with the increase of the permeability of the porous medium, magnetic parameter, magnetic diffusivity number and Hall parameter, while it decreases with the increase of $(\alpha)$.

5. The average and local Nusselt number decreases with the increase of the Dufour number, heat generation or absorption parameter and Eckert number whereas reverse effects have been observed with the increse of the Prandtl number.

6. The average and local Sherwood number decreases with the increase of the Prandtl number and Soret number while reverse effects have been found with the increse of the heat generation or absorption parameter, Schmidt number and chemical reaction parameter.

The findings of this investigation may be useful for the study of movement oil or gas and water through the reservoirs of an oil or gas field, in the migration of underground water or oil as well as in the filtration and water purification processes. These results may also be useful for plasma studies as well as in power engineering, geothermal energy extraction, geophysics and astrophysics.

\section{Nomenclature}

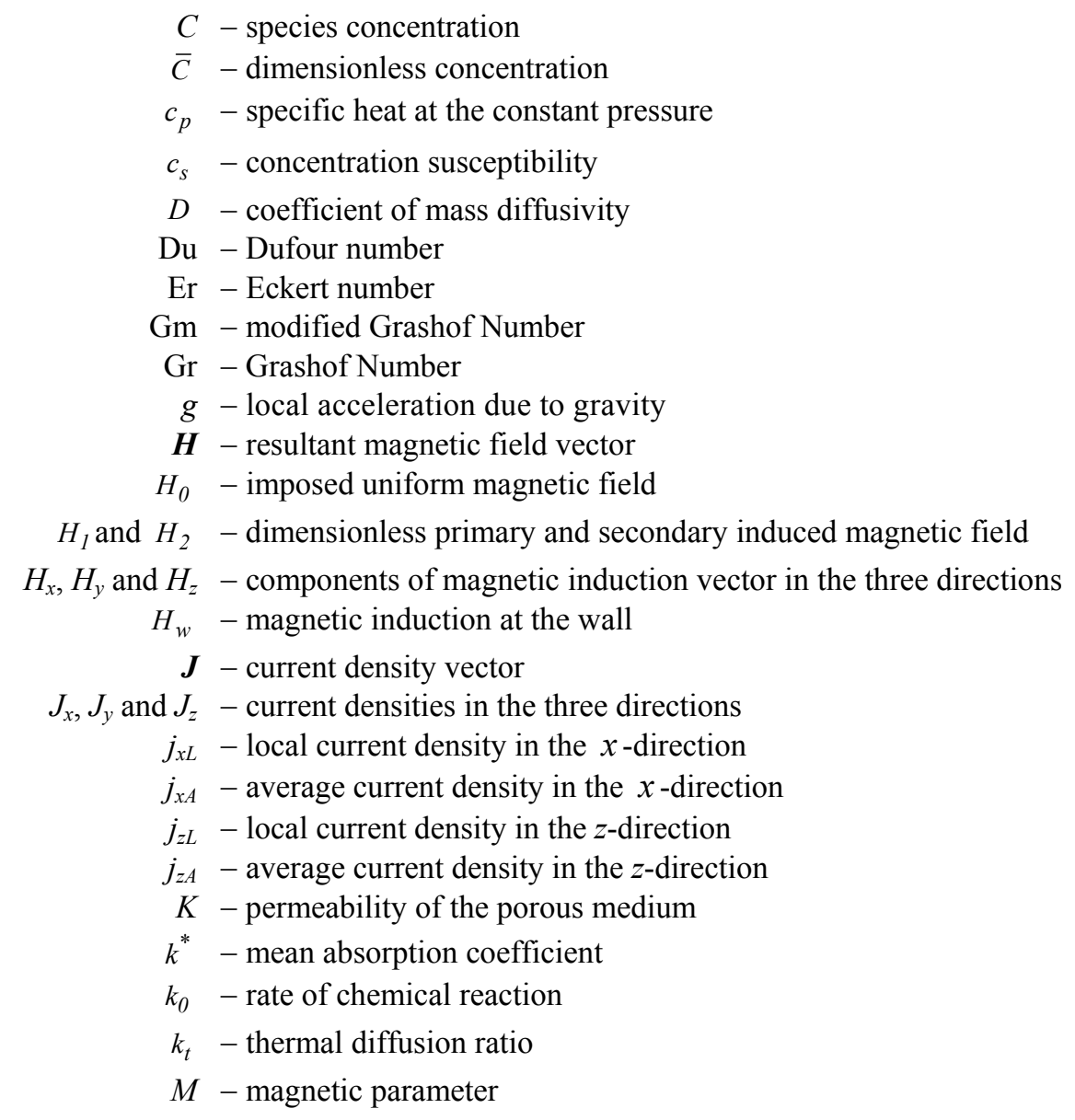




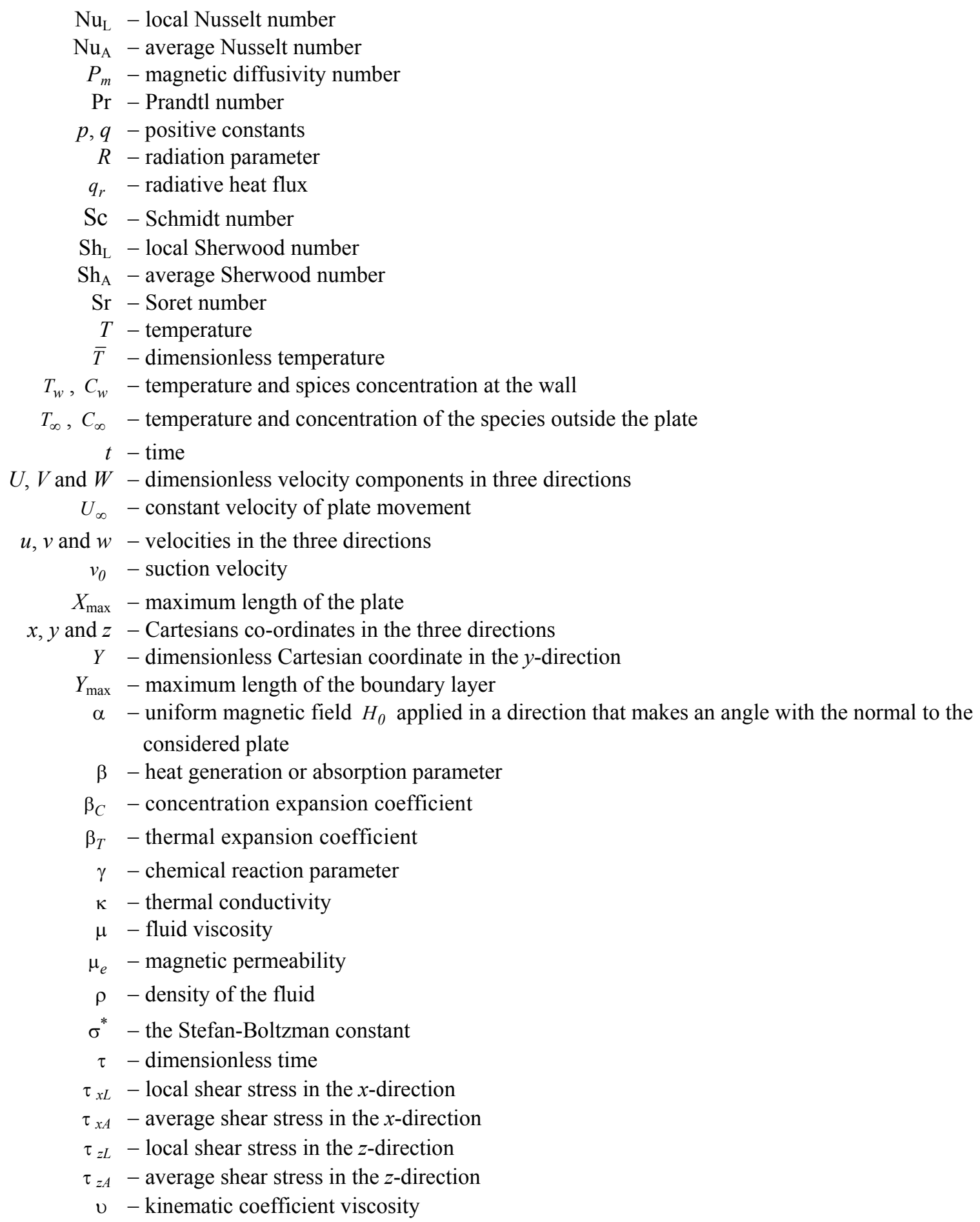

\section{References}

[1] Cowling T.G. (1957): Magnetohydrodynamics. - New York: Interscience Publications.

[2] Gebhart B. and Pera L. (1971): The nature of vertical natural convection flows resulting from the combined buoyancy effects of thermal and mass diffusion. - International Journal of Heat and Mass Transfer, vol.14, No.12, pp.2025-2050. 
[3] Ram P.C. (1988): Unsteady MHD free-convective flow through a porous medium with Hall currents. Astrophysics and Space Science, vol.149, pp.171-174.

[4] Sattar M.A. and Alam M.M. (1995): MHD free convective heat and mass transfer flow with Hall current and constant heat flux through a porous medium. - Indian J. Pure Appl. Math., vol.26, No.2, pp.157-167.

[5] Aydin O. and Kaya A. (2008): Radiation effect on MHD mixed convection flow about a permeable vertical plate. - Heat Mass Transfer, vol.45, pp.239-246.

[6] Shateyi S., Mosta S.S. and Sibanda P. (2010): The effects of thermal radiation, Hall currents, Soret and Dufour on MHD flow by mixed convection over a vertical surface in porous media. - Mathematical Problems in Engineering, Article ID 627475.

[7] Olanrewaju P.O. and Gbadeyan J.A. (2010): Effects of Soret, Dufour, chemical reaction, thermal radiation and volumentric heat generation/absorption on mixed convection stagnation point flow on an iso-thermal vertical plate in porous media. - Pacific Journal of Science and Technology, vol.11, No.2, pp.1-12.

[8] Aurangzaib and Shafie S. (2011): Effects of Soret and Dufour on unsteady MHD flow by mixed convection over a vertical surface in porous media with internal heat generation, chemical reaction and Hall current. Canadian Journal on Science and Engineering Mathematics, vol.2, No.4, pp.153-162.

[9] Ahmed T. and Alam M.M. (2013): Finite difference solution of MHD mixed convection flow with heat generation and chemical reaction. - Proceedia Engineering, vol.56, pp.149-156.

[10] Chaudhary R.C. and Sharma B.K. (2006): Combined heat and mass transfer by laminar mixed convection flow from a vertical surface with induced magnetic field. - J. Appl. Phys., vol.99, No.3, pp.034901.

[11] Alam M.M., Islam M.R., Wahiduzzaman M. and Rahman F. (2012): Unsteady heat and mass transfer by mixed convection flow from a vertical porous plate with induced magnetic field, constant heat and mass fluxes. Journal of Energy, Heat and Mass Transfer, vol.34, pp.193-215.

[12] Haque M.M., Alam M.M., Ferdows and Al-Mdallal Q.M. (2013): Numerical simulation and stability analysis on MHD free convective heat and mass transfer unsteady flow through a porous medium in a rotating system with induced magnetic field. - International Journal of Applied Electromagnetic and Mechanics, vol.41, pp.121141.

[13] Carnahan B., Luther H.A. and Wilkes J.O. (1969): Applied Numerical Methods. - New York: John Wiley and Sons.

[14] Brewster M.Q. (1992): Thermal Radiative Transfer and Properties. - New York: USA: John Wiley and Sons Inc.

Received: September 7, 2017

Revised: September 27, 2018 